\title{
Large Eddy Simulation of a Bluff Body Stabilized Lean Premixed Flame
}

\author{
A. Andreini, C. Bianchini, and A. Innocenti \\ Department of Industrial Engineering, University of Florence, Via di Santa Marta 3, 50139 Florence, Italy \\ Correspondence should be addressed to A. Andreini; antonio.andreini@htc.de.unifi.it
}

Received 28 June 2013; Revised 5 October 2013; Accepted 7 October 2013; Published 14 January 2014

Academic Editor: Eliseo Ranzi

Copyright (C) 2014 A. Andreini et al. This is an open access article distributed under the Creative Commons Attribution License, which permits unrestricted use, distribution, and reproduction in any medium, provided the original work is properly cited.

The present study is devoted to verify current capabilities of Large Eddy Simulation (LES) methodology in the modeling of lean premixed flames in the typical turbulent combustion regime of Dry Low $\mathrm{NO}_{x}$ gas turbine combustors. A relatively simple reactive test case, presenting all main aspects of turbulent combustion interaction and flame stabilization of gas turbine lean premixed combustors, was chosen as an affordable test to evaluate the feasibility of the technique also in more complex test cases. A comparison between LES and RANS modeling approach is performed in order to discuss modeling requirements, possible gains, and computational overloads associated with the former. Such comparison comprehends a sensitivity study to mesh refinement and combustion model characteristic constants, computational costs, and robustness of the approach. In order to expand the overview on different methods simulations were performed with both commercial and open-source codes switching from quasi-2D to fully 3D computations.

\section{Introduction}

The emission reduction, especially of $\mathrm{NO}_{x}$, has been the major driver for gas turbine development in the last decades. One of the most promising gas turbine combustion technologies to respect the strict legislative limits on pollutant emissions is the adoption of lean premixed flame. In the fields of combustion science and engineering, CFD calculations are now truly competitive with experiments and theory, as a research tool to produce detailed and multiscale information about combustion processes and play a crucial role in the design of environment-friendly devices. In particular, gas turbine combustion modeling, involving the interaction of many complex physical processes such as turbulent mixing and chemical reactions, comprises a wide range of computational and modeling challenges [1]. In this context LES is one of the most promising techniques as it allows a detailed resolution of the flow field and turbulent mixing phenomena.

An axisymmetric bluffbody stabilized flame, reproducing typical lean premixed gas turbine combustor's conditions, has been numerically studied, under adiabatic conditions, with the commercial code ANSYS Fluent vers.14.0, using LES coupled with the progress variable $(c)$ approach closed with Zimont Turbulent Flame Speed Closure (TFC) [2].

Numerical settings, mesh and time step sensitivity analysis are at first performed on a quasi-2D test case, representing a 5-degree slice of the complete geometry. Successively the fully $3 \mathrm{D}$ geometry has been simulated varying the combustion model constant controlling the source term in progress variable transport equation (Product Formation Rate, PFR). Finally an improved mesh arrangement in the region near the bluffbody has been generated in order to investigate the influence of mesh refinement on smallest structures wrinkling the flame front and PFR.

LES simulations have also been performed with the open-source CFD code OpenFOAM both using its native modelling for premixed combustion as well as an in-house developed solver with turbulent flame speed closure similar to that available in ANSYS Fluent. This second test permitted separating the discrepancies due to different combustion models by those related to numerics.

Results are compared, in terms of mean velocity and mean temperature, with available experimental data, published LES calculations results found in literature, and RANS simulations performed on the same test case. A computational cost 


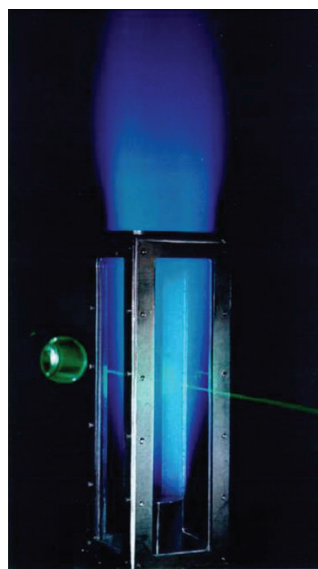

\begin{tabular}{lr}
\hline$V_{\text {ref }}(\mathrm{m} / \mathrm{s})$ & 15 \\
$\phi(-)$ & 0.586 \\
$D(\mathrm{~mm})$ & 44.45 \\
$T(\mathrm{~K})$ & 294.0 \\
$P(\mathrm{bar})$ & 1.0 \\
$\mathrm{TU}(\%)$ & 24.0 \\
$L_{T}(\mathrm{~mm})$ & 3.6 .0 \\
$\operatorname{Re}(D)(-)$ & 43400 \\
$\operatorname{Re}_{T}(-)$ & 625 \\
\hline
\end{tabular}

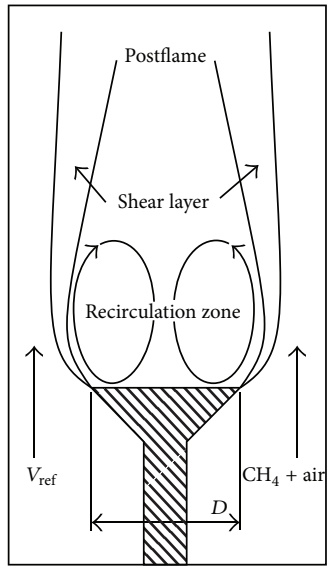

FIGURE 1: Vanderbilt combustor and reference conditions.

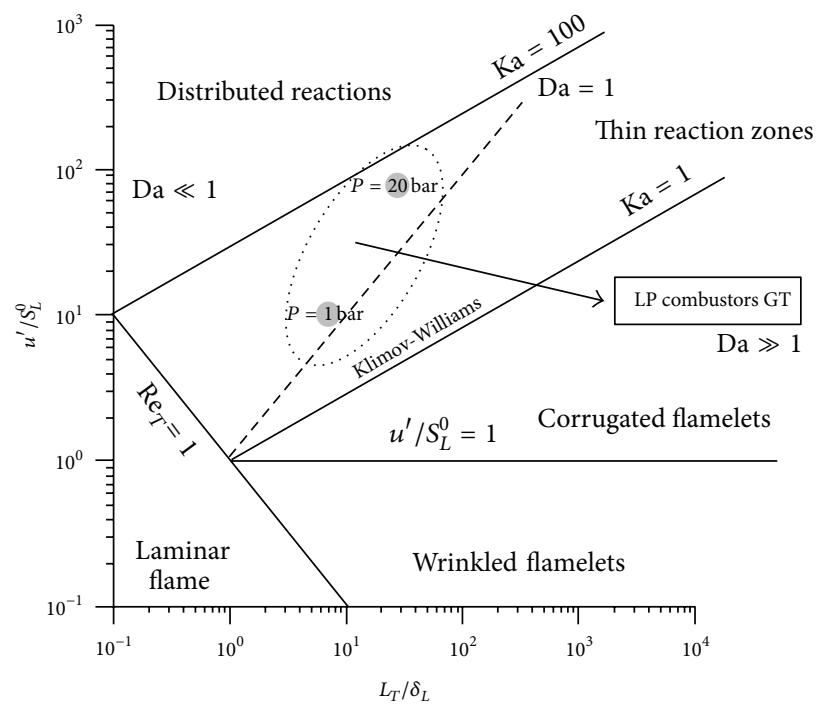

FIGURE 2: Comparison of turbulent combustion regime of VDB and typical lean premixed gas turbine combustors.

TABLE 1: Mesh sensitivity analysis data.

\begin{tabular}{lcc}
\hline Mesh & Number of elements & $\beta_{\max }$ \\
\hline A & 26727 & 0.2 \\
B & 62160 & 0.13 \\
C & 153276 & 0.11 \\
\hline
\end{tabular}

TABle 2: Performed runs.

\begin{tabular}{lcccc}
\hline & RANS & LES-Zim-FL & LES-Zim-FL & LES-Zim-FL \\
& Zim-FL & M1-A & M2-A & M2-2A \\
\hline Mesh elements & 915000 & 915000 & 2400000 & 2400000 \\
A constant & 0.52 & 0.52 & 0.52 & 1.04 \\
\hline
\end{tabular}

analysis has been finally carried out to provide feasibility guidelines for future works.

1.1. Specific Objectives. The main objective of this work is to test different reactive LES implementations in order to understand their potentials and limitations and to compare them with RANS simulations in terms of mean results, calculation time, and modeling accuracy gains.

\section{Turbulent Premixed Flames Modeling}

Premixed flames fronts are generally very thin with a thickness $\delta$ in the range of $0.1 \div 1 \mathrm{~mm}$, which is, in many cases, smaller than the filter width $\Delta$ used in LES. In a LES context, one approach to model such a flame is to ignore its internal structure and the detailed chemical kinetics and to represent the combustion occurring at the flame front in terms of a progress variable ( $\mathrm{PV}$ or $c$ ), which varies from 0 (fresh reactants) to 1 (burnt gases). Possible definitions of the progress variable can be provided in terms of reduced temperature or reduced fuel mass fraction, given by

$$
c=\frac{T-T_{u}}{T_{b}-T_{u}} \quad c=\frac{Y-Y_{u}}{Y_{b}-Y_{u}}
$$



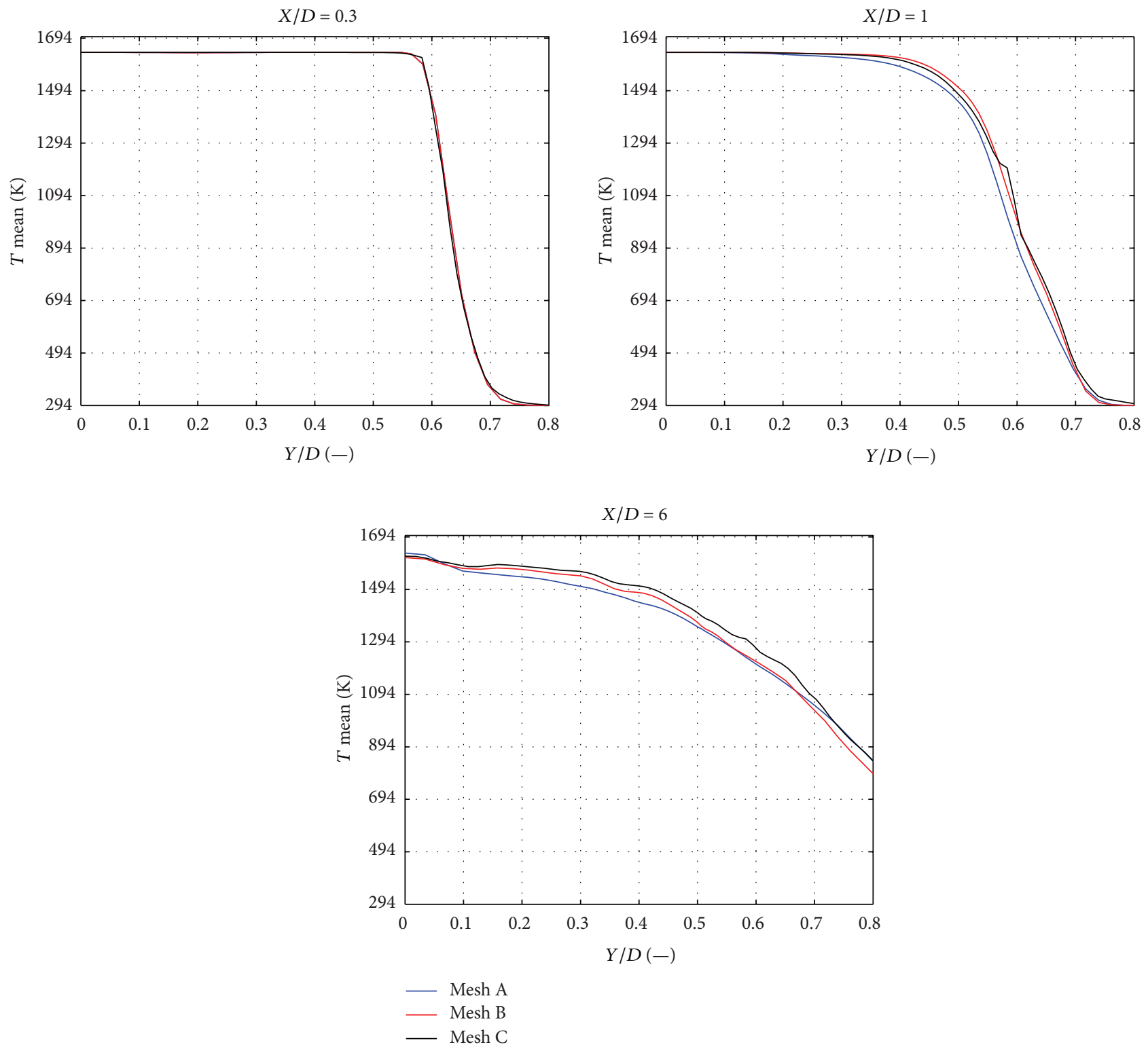

FIGURE 3: Mean temperature profiles resulting from mesh size sensitivity analysis.

where $T, T_{u}$, and $T_{b}$ are, respectively, local, unburnt, and burnt gases temperature, while $Y, Y_{u}$, and $Y_{b}$ are local, unburnt, and burnt species mass fractions. In case of Lewis number $L e=1$, and if the constant pressure specific heat $c_{p}$ is retained the same for both states (burnt and unburnt), it is possible to demonstrate that the two definitions coincide [4].

Adopting this approach in a LES context, a Favre-filtered transport equation for $c(2)$ is solved in conjunction with the filtered momentum equations:

$$
\frac{\partial \bar{\rho} \widetilde{c}}{\partial t}+\frac{\partial\left(\bar{\rho} \widetilde{u_{l}} \widetilde{c}\right)}{\partial x_{i}}=\frac{\partial}{\partial x_{i}}\left(\left(\overline{\rho D}+\frac{\mu_{s g s}}{S c_{T}}\right) \frac{\partial \widetilde{c}}{\partial x_{i}}\right)+\overline{\rho S}_{c}
$$

In ANSYS Fluent implementation, the subfilter scalar term is modeled by means of gradient based assumption with the turbulent Schmidt number $S c_{T}$ set constant to 0.7, while subgrid scale viscosity is obtained from the standard Smagorinsky model. Source term is modeled using Zimont's TFC and set proportional to the gradient of the filtered progress variable and to a turbulent flame speed $S_{T}$ that depends on the physical-chemical characteristics of the fuel mixture through its laminar flame speed $S_{l}$ and on the local turbulence at the subgrid level:

$$
\overline{\rho S_{c}}=\overline{\rho_{u}}|\nabla \widetilde{c}| \overline{S_{T}}=|\nabla \widetilde{c}|\left(A{\overline{u^{\prime}}}^{3 / 4} S_{l}^{1 / 2} \chi^{-1 / 4} l_{t}^{1 / 4}\right),
$$

where $u^{\prime}=C_{s} \Delta|\bar{S}|, l_{t}=C_{s} \Delta, C_{s}=0.1$, and $\bar{S}$ the resolved strain tensor and $\chi$ the thermal diffusivity. The model constant $A$ is empirical and the suggested value is 0.52 for most hydrocarbon fuels. To take flame stretching into account, the source term for the progress variable is multiplied by a stretch factor $G$, calculated as a function of 

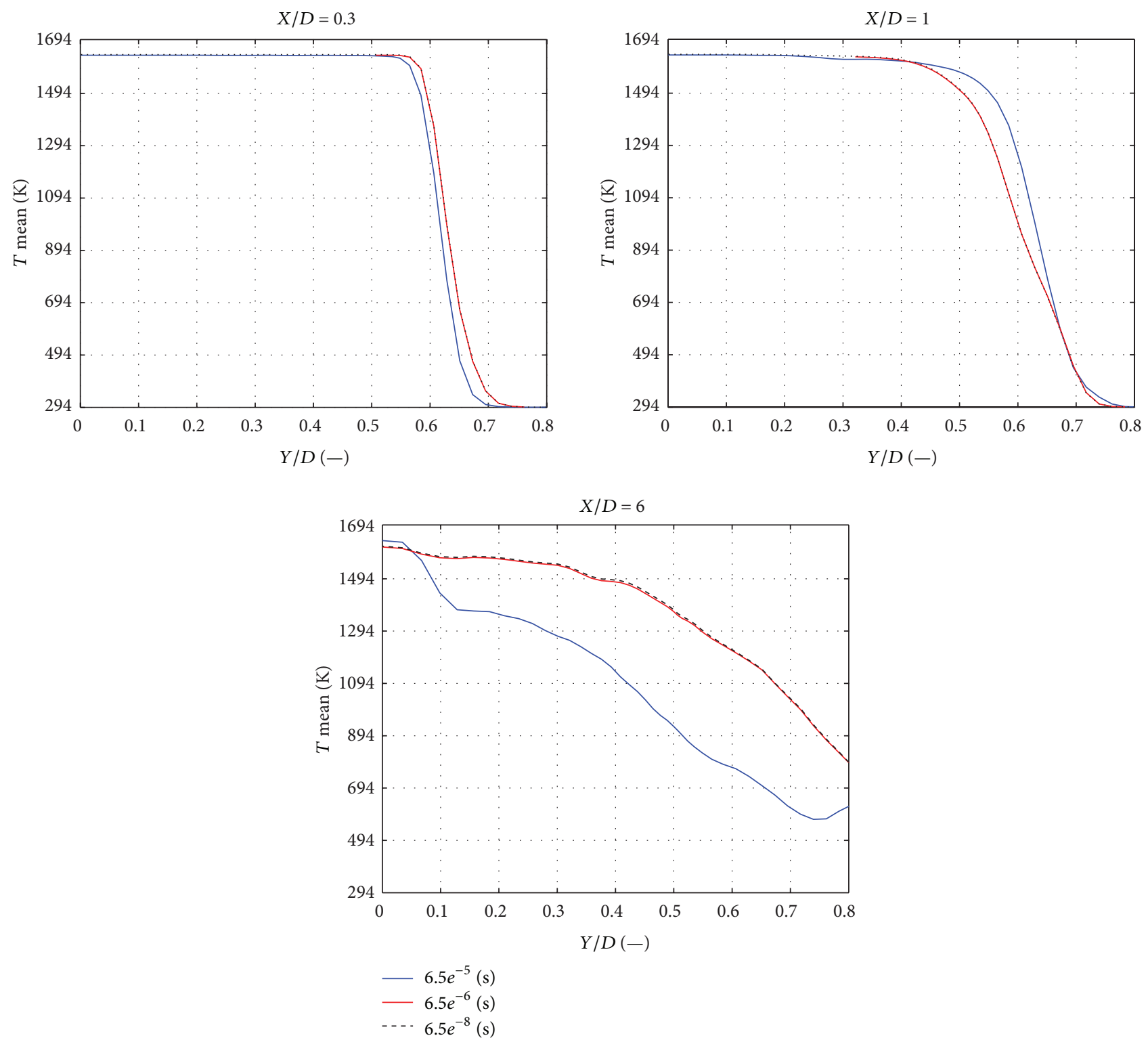

FIGURE 4: Mean temperature profiles resulting from time step size sensitivity analysis.

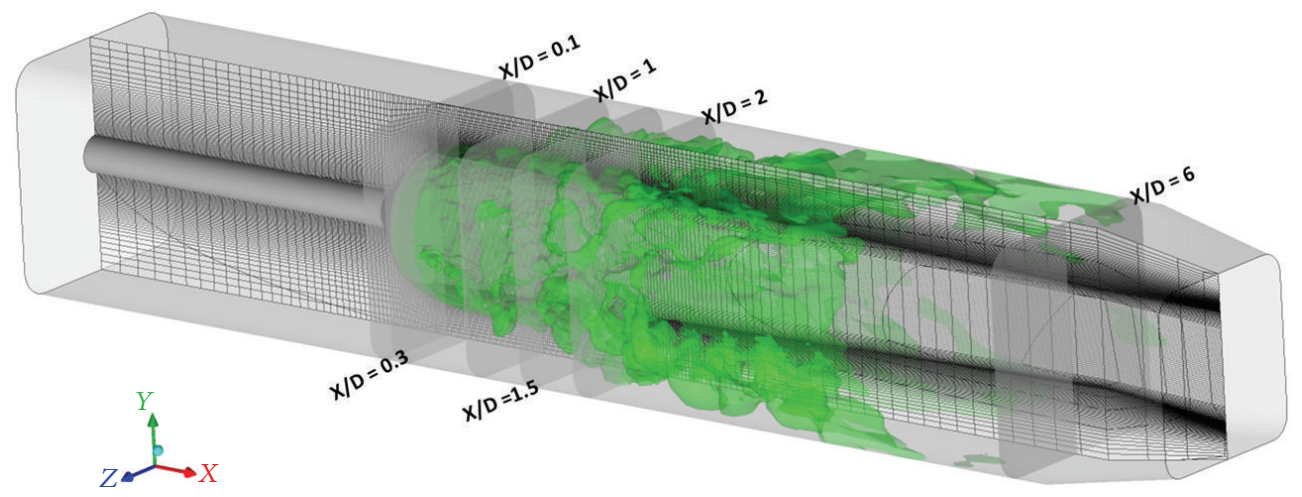

Figure 5: Simulated domain, sample planes, and a mesh plane. 


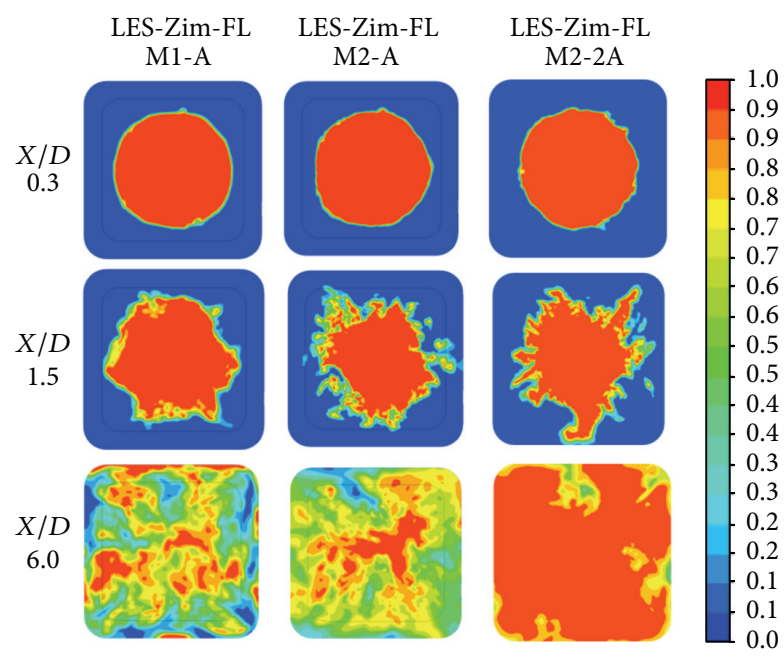

FIGURE 6: Instantaneous progress variable contours at three axial locations.

the turbulent dissipation rate $(\varepsilon)$, the subgrid scales properties, and the critical strain rate $\left(g_{\mathrm{cr}}\right)$ that is by default set to a high value so that no flame stretch occurs [5]. No flame speed reduction has been introduced in the wall proximity to account for wall turbulence damping. RANS formulation of the combustion model has been adopted when performing such simulations together with a RNG $k-\varepsilon$ turbulence model.

The OpenFOAM available formulation for flame front propagation within premixed combustion regime is instead based on solving a transport equation for the Favre-filtered regress variable $b=1-c$ :

$$
\frac{\partial \bar{\rho} \tilde{b}}{\partial t}+\frac{\partial\left(\bar{\rho} \tilde{u_{l}} \tilde{b}\right)}{\partial x_{i}}=\frac{\partial}{\partial x_{i}}\left(\left(\overline{\rho D}+\frac{\mu_{s g s}}{S c_{T}}\right) \frac{\partial \tilde{b}}{\partial x_{i}}\right)-\overline{\rho S}_{c} .
$$

The $b$-equation source term is modeled as

$$
\rho S_{c}=\rho_{u}|\nabla b| \Xi S_{l} .
$$

With turbulent to laminar flame speed ratio $\Xi$ (also referred as $\mathrm{Xi}$ ) solved by means of a transport equation as proposed in [6]. However, in order to stress the differences only due to the different numerics available in the two codes, the same Zimont closure as proposed in Fluent was also implemented and tested in OpenFOAM, maintaining the same framework based on the regress variable.

Sub-grid fluctuations model is based on a transport equation for the sub-grid turbulent kinetic energy as proposed by Yoshizawa [7] and Menon et al. [8] which is accredited for a better prediction of nonequilibrium zones compared to typical algebraic sgs models.

\section{Test Case Description}

A classical way of stabilizing a turbulent premixed flame consists in generating a large recirculation where the low velocity zone permits flame anchoring with hot gasses recirculating in proximity of the fresh mixture and continuously igniting the reactive stream. A way of achieving such flow distribution is using a bluff body inserted in the mixture stream whose low pressure back side guarantees the driving force for the large recirculation bubble. Turbulence enhances mixing downstream of the bluff body, anchoring the flame in an unsteady manner. Combustors with bluff body flame holders, like that analyzed in this work, are characterized by a shear layer where vortices are shed due to Kelvin-Helmholtz instability [9]. This shear layer separates the region of highspeed fresh mixture from the wake region of lower speed (recirculation zone) with hot products.

The test case adopted is an experimental burner studied at Vanderbilt University, Tennessee, USA (VDB), which allows reproducing all the effects of the turbulent mixing and turbulence-combustion kinetic interaction to be observed and investigated at the same time.

Figure 1 shows a picture of the flame realized by VDB combustor. The flame is confined in a square-section duct with quartz walls. A $45^{\circ}$ conical bluff body, characterized by a diameter $D$, is located coaxially to stabilize a premixed methane-air flame.

This work will refer, for calculation comparisons, to Nandula's measurements [10] which include laser Doppler anemometry velocity and turbulence data as well as temperature profiles based on Rayleigh scattering.

Reference values of velocity $V_{\text {ref }}$, turbulent intensity TU, an integral scale length $L_{T}$ must be considered at the test section, immediately before recirculation zone. Equivalence ratio $\phi=0.586$, representative of a lean premixed combustor flame, is considered.

In order to point out the capacity of this test case to be representative of turbulent combustion flames of gas turbine lean premixed combustors, an attempt was made to locate its combustion regime in a classical spectral Borghi diagram following the classification proposed by Peters [11]. According to Peters, it is possible to extend the validity of flamelet regime also when Karlovitz number $\left(\mathrm{Ka}=\left(\delta_{L} / L_{k}\right)^{2}\right)$ is slightly greater than unity $(\mathrm{Ka}<100)$. In this regime, called thin reaction zone, the scale of smallest vortexes $\left(L_{k}\right)$ is still greater than the thickness of laminar flamelet inner layer $\left(1 / 10 \delta_{L}\right.$, being $\delta_{L}$ overall laminar flame thickness), which is the reaction zone core of laminar structure. Figure 2 reports the expected locations on the diagram of a typical actual gas turbine lean premixed combustor (operating at 20 bar) and the VDB bluff body (operating at ambient pressure).

\section{Domain Definition and Numerical Methods}

4.1. Boundary Conditions. At inlet section a constant velocity profile is imposed with free-stream turbulence set to $24 \%$ and integral length scale to $3.6 \mathrm{~mm}$ in RANS simulation, while LES method employs synthetic turbulence generation methods. ANSYS Fluent implements the so-called "Vortex Method" [5] which is based on a Lagrangian form of the vorticity equation to provide perturbations on a given mean profile. On the other hand, only a quite basic synthetic turbulent generator based on white noise random fluctuation superposition is available in OpenFOAM. Hence, in order to provide realistic flow conditions within the upstream feeding 


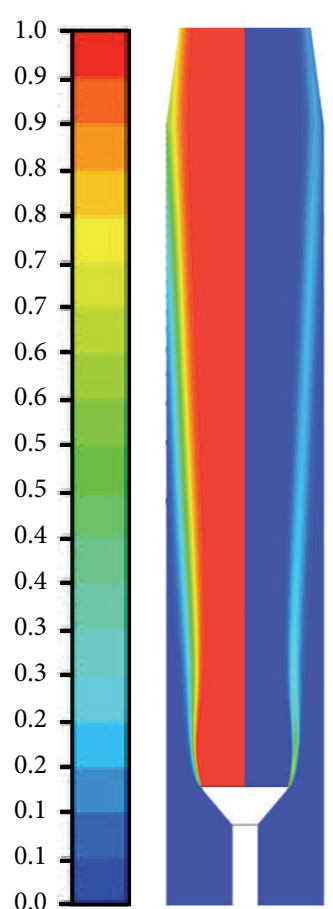

RANS-Zim-FL
Progress variable $c \mid$ PFR

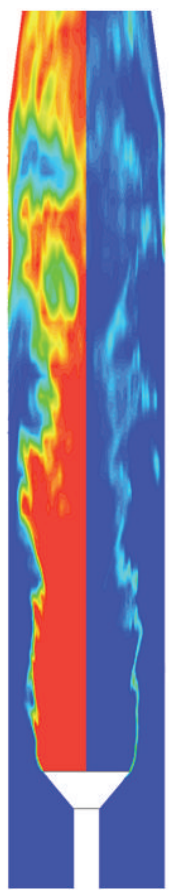

LES-Zim-FL M1-A

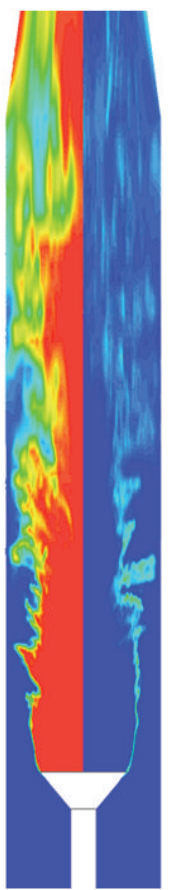

LES-Zim-FL M2-A

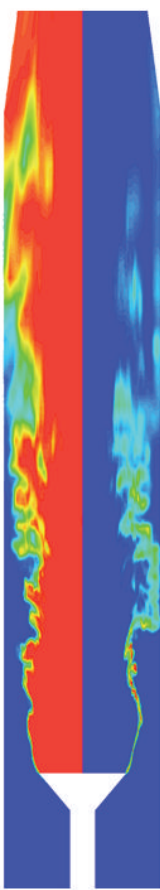

LES-Zim-FL

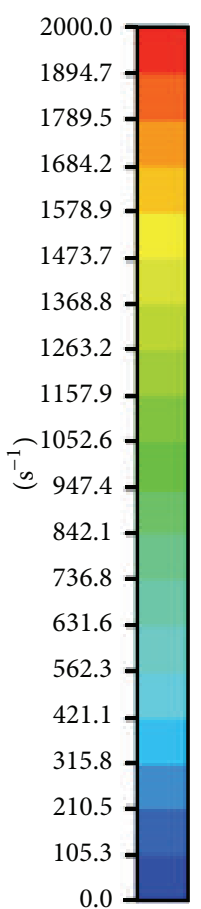

0.0

FIGURE 7: Instantaneous progress variable and PFR contours (longitudinal view).
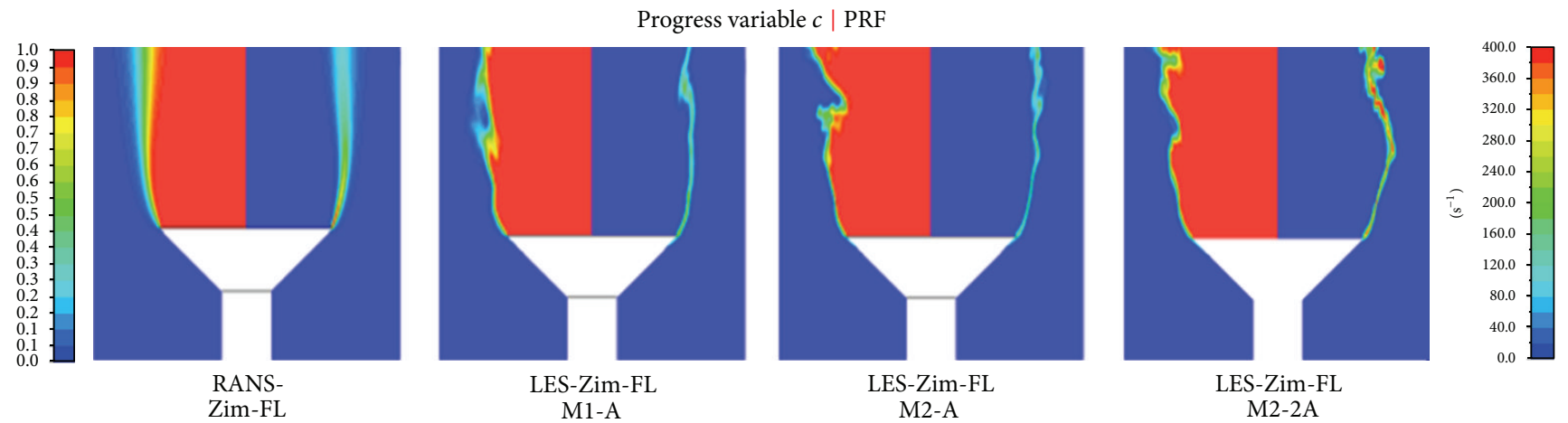

FIGURE 8: Instantaneous progress variable and PRF (bluff body details).

channel, an autorecycling technique [12] was exploited. Such technique consists in the mapping of self-developed turbulent fields back on the inflow introducing feedback mechanism to guarantee prescribed mean profiles. An approach capable of dealing with arbitrarily shaped mesh was implemented and employed in this context. Uniform pressure is assigned on the outlet neglecting all types of reflections of pressure or entropy waves, while bluffbody and duct walls are considered adiabatic realizing no-slip conditions.

4.2. Fluent Numerical Settings. Iterative time advancement is used in Fluent with segregated SIMPLEC algorithm to solve internal iteration pressure-velocity coupling. For pressure equation, a linear discretization is chosen as it leads to less numerical dissipation, resulting in a more refined flame front reproduction compared with other discretization schemes. A central differencing scheme based on a deferred approach is used for momentum and progress variable equations as it enhances stability respect to the purely linear scheme. The choice of the numerical schemes used has been made after preliminary analysis aimed at defining the best setting in terms of accuracy, robustness, and computational efficiency [13].

The thermophysical properties of the air methane mixture with $\phi=0.586$ have been determined using CHEMKIN libraries. In particular unburnt mixture density $\rho_{u}$ at reference temperature of $294 \mathrm{~K}$ was found to be $1.115 \mathrm{~kg} / \mathrm{s}$, thermal diffusivity $1.0 \mathrm{E}-5 \mathrm{~m}^{2} / \mathrm{s}$, and adiabatic flame temperature 


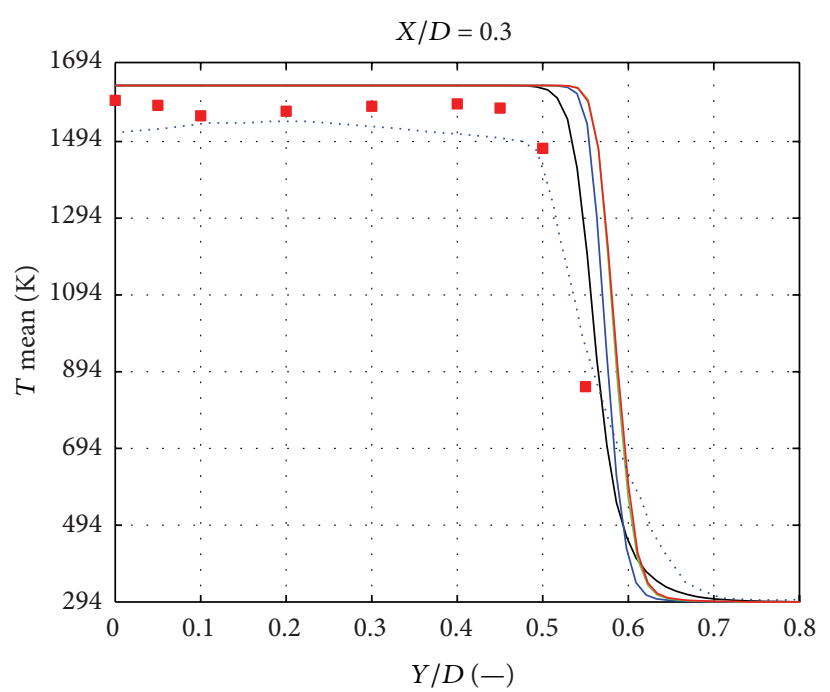

- $\operatorname{Exp}$

- RANS-Zim-FL

— LES-Zim-FL-M1-A

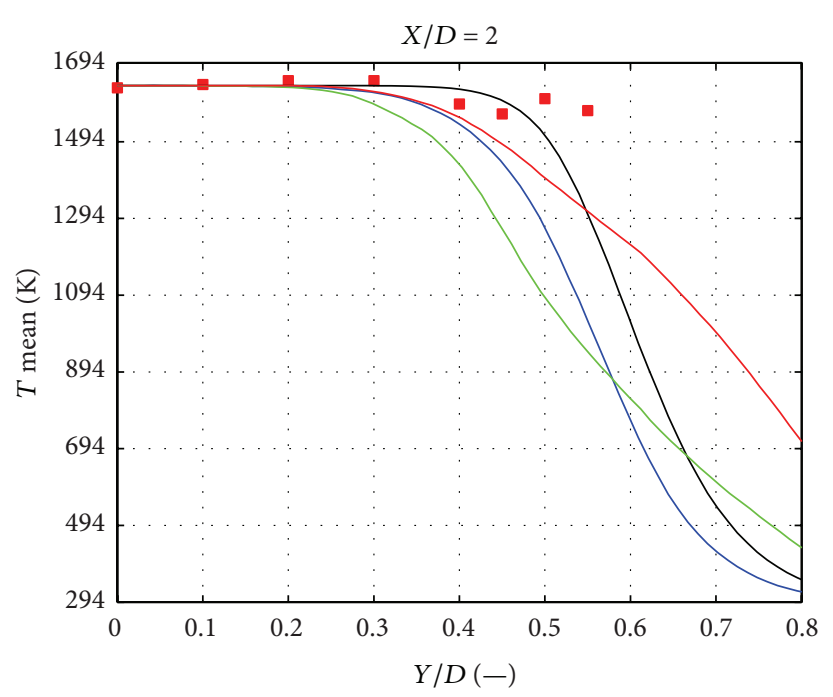

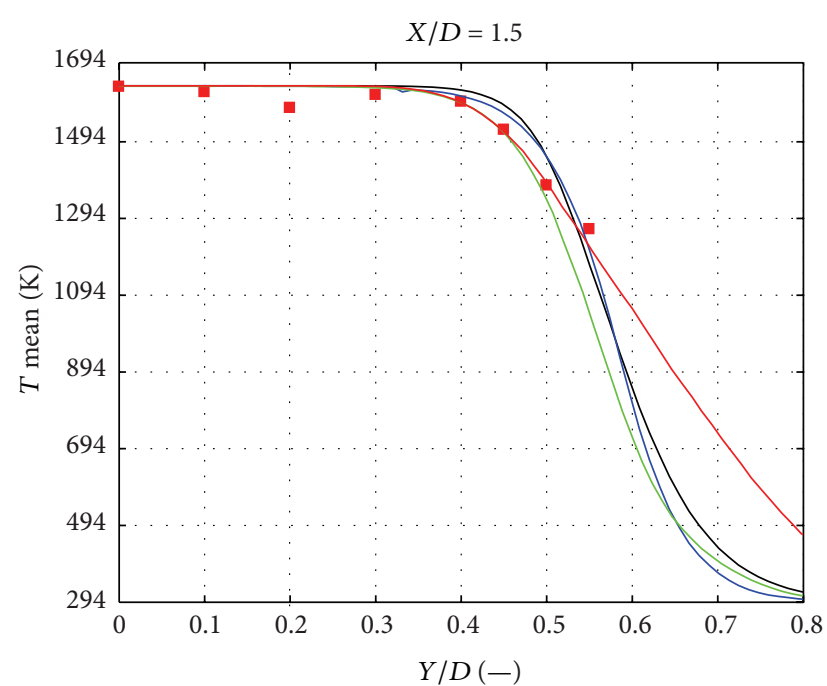

- Exp - LES-Zim-FL-M2-A

- RANS-Zim-FL _ LES-Zim-FL-M2-2A

— LES-Zim-FL-M1-A

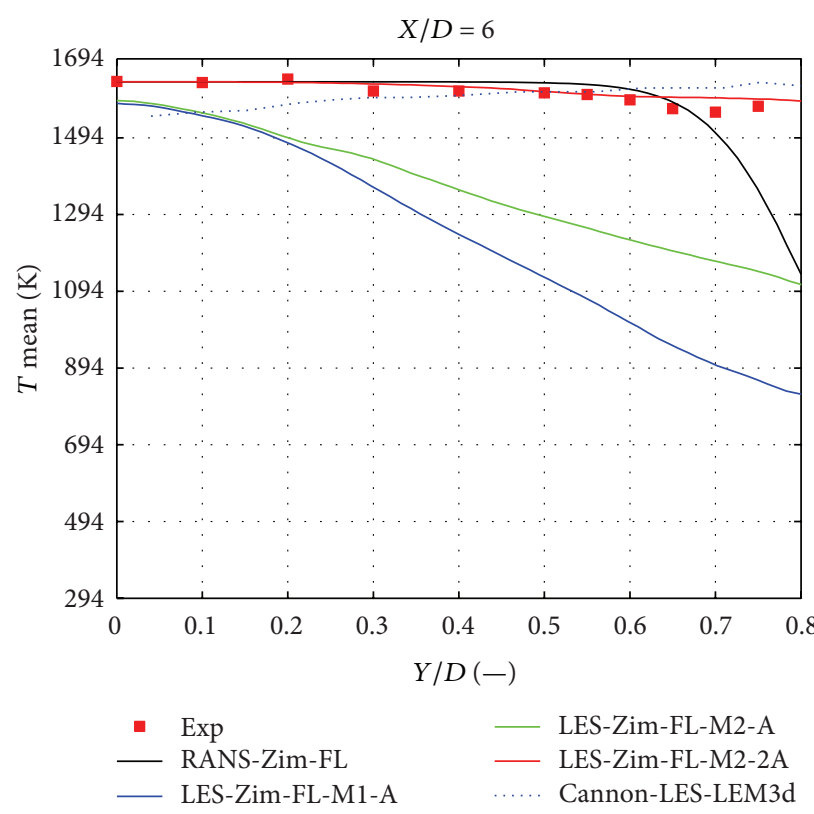

FIgURE 9: Mean temperature profiles at different axial locations.

$1640.00 \mathrm{~K}$. For TFC model the unstretched laminar flame speed was assumed equal to $0.11[\mathrm{~m} / \mathrm{s}]$.

4.3. OpenFOAM Numerical Settings. Besides the already mentioned combustion and subgrid model, other numerical and modeling strategies differ among Fluent and OpenFOAM. First of all the solver in this case considers nonadiabatic conditions: energy equation is solved, and JANAF table is used to compute thermophysical properties of the mixture. Sutherland law is employed for the transport properties of both burnt and unburnt gases.

The solver follows a classical OpenFOAM segregated method called PIMPLE which is based on a PISO loop nested within a SIMPLE loop to solve the pressure-velocity coupling. Due to the small time step employed in the analysis, the solver uses only 3 internal corrector steps to achieve coupling between continuity and momentum equations and 2 external with high relaxation factors (i.e., >0.8). Both convective and diffusive fluxes are discretized following purely linear schemes to maximize accuracy as necessary for LES, except for the regress variable equation where a filtered scheme is adopted to guarantee stability; time advancement is achieved by means of implicit Euler scheme.

Linear algebra exploits acceleration with the use of Diagonal Incomplete LU factorization to precondition BiConjugate gradient solver. Matrices are solved with a relative tolerance 


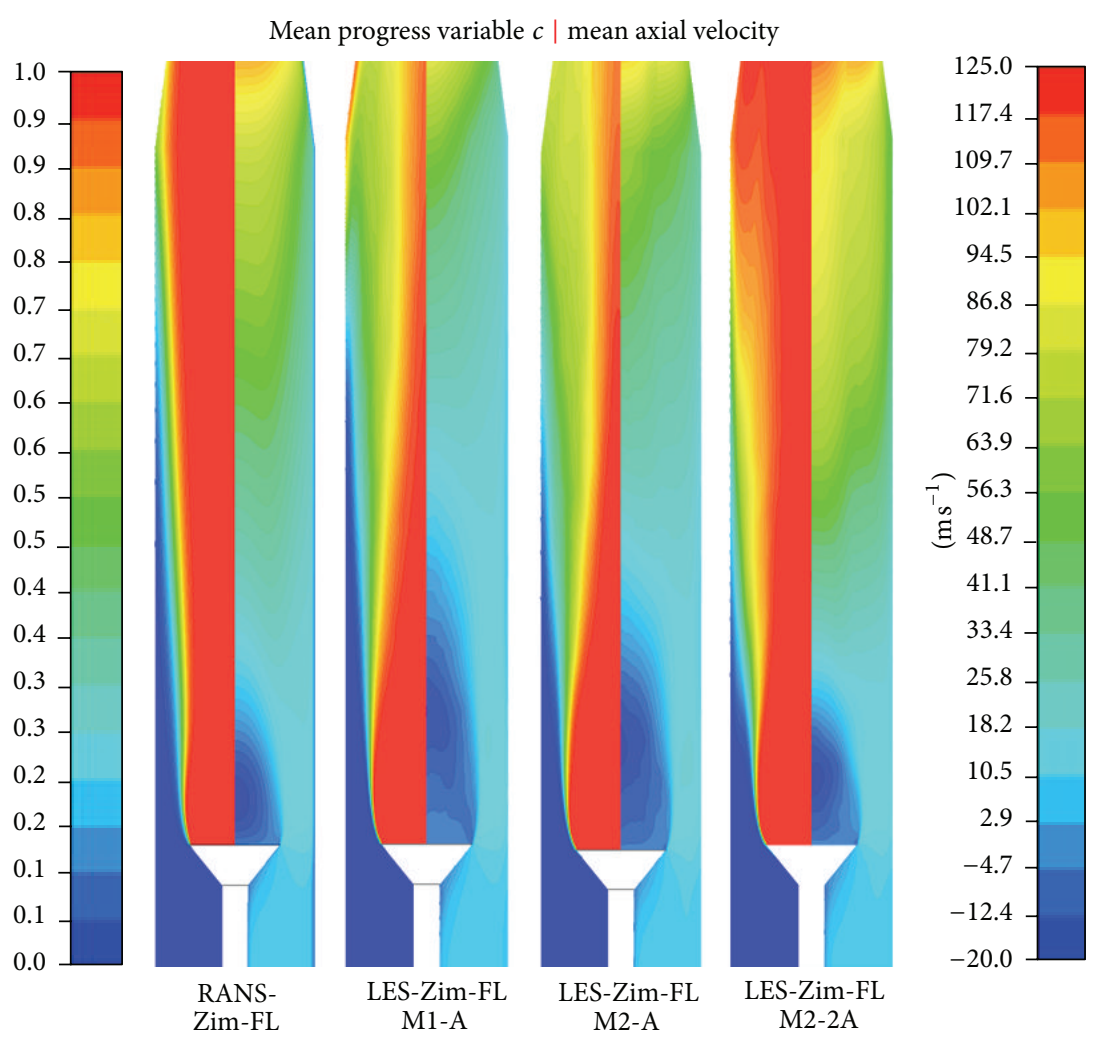

FIGURE 10: Mean progress variable and axial velocity contours (longitudinal view).

of 0.1 for the inner iterations and $1 e^{-7}$ absolute tolerance for the last one.

4.4. Computational Mesh. All finite volume grids have been realized with the commercial tool ICEM-CFD V.13.0. Multiblock structured topology is employed. A progressive refinement of the mesh is realized in the zone where the shear layer is present in order to well resolve the local turbulent structures. Their influence is in fact crucial for an accurate reproduction of the combustion phenomena evolution. A coarsening of the mesh elements is instead accepted in the final part of the domain towards outlet section.

\section{Results and Discussion}

5.1. Quasi-2D Preliminary Study. Before simulating the complete $3 \mathrm{D}$ test case, it has been considered useful to calibrate the procedure on a simplified and less onerous quasi-2D test case. Even though two-dimensional behaviour should never be assumed in case of direct resolution of turbulent structures (LES or DNS) the axisymmetric geometry allows reducing the problem to an equivalent angular sector $\left(5^{\circ}\right)$.

It should be noticed that tangential fluctuations are considerably less intense and do not produce significant turbulent mixing and combustion enhancement in this case. In the quasi-2D case relevant phenomena are then slightly altered and the main features can still be observed. This means that mesh and time step sensitivity considerations may be retained valid also for the $3 \mathrm{D}$ case.
Periodic conditions are imposed on the side boundaries, limiting the tangential fluctuations but substantially reproducing the main flame development. Mesh sensitivity analysis and time step validation have been conducted to establish the optimal numerical setting, which is then applied to the complete 3D calculations.

A criterion for a first estimation of the mesh refinement can be found in [14], according to whom the filter width $\Delta$ should be dimensioned relatively to the mean turbulent flame thickness $\delta_{T}$ by

$$
\Delta \cong \beta \delta_{T}
$$

proposing $\beta \leq 0.09$ for a first estimation. To verify this criteria, it is necessary to define and provide a value for $\delta_{T}$ that is absolutely difficult. It is decided to estimate $\delta_{T}$ a posteriori from RANS solution as follows:

$$
\delta_{T}=\frac{\text { Volume }_{0.1<c<0.9}}{\text { Area }_{c=0.5}},
$$

that is the ratio between the volume included by the two isosurfaces at progress variable $c=0.1$ and $c=0.9$ and the area of the isosurface at $c=0.5$ bearing in mind that RANS flame front is thicker than the instantaneous LES one.

The maximum values of $\beta$ for the three meshes are reported in Table 1 together with the number of elements. The coarsest mesh did not allow correctly reproducing the smallest structures wrinkling the flame front if compared with the two more refined meshes. Such effect does not affect 

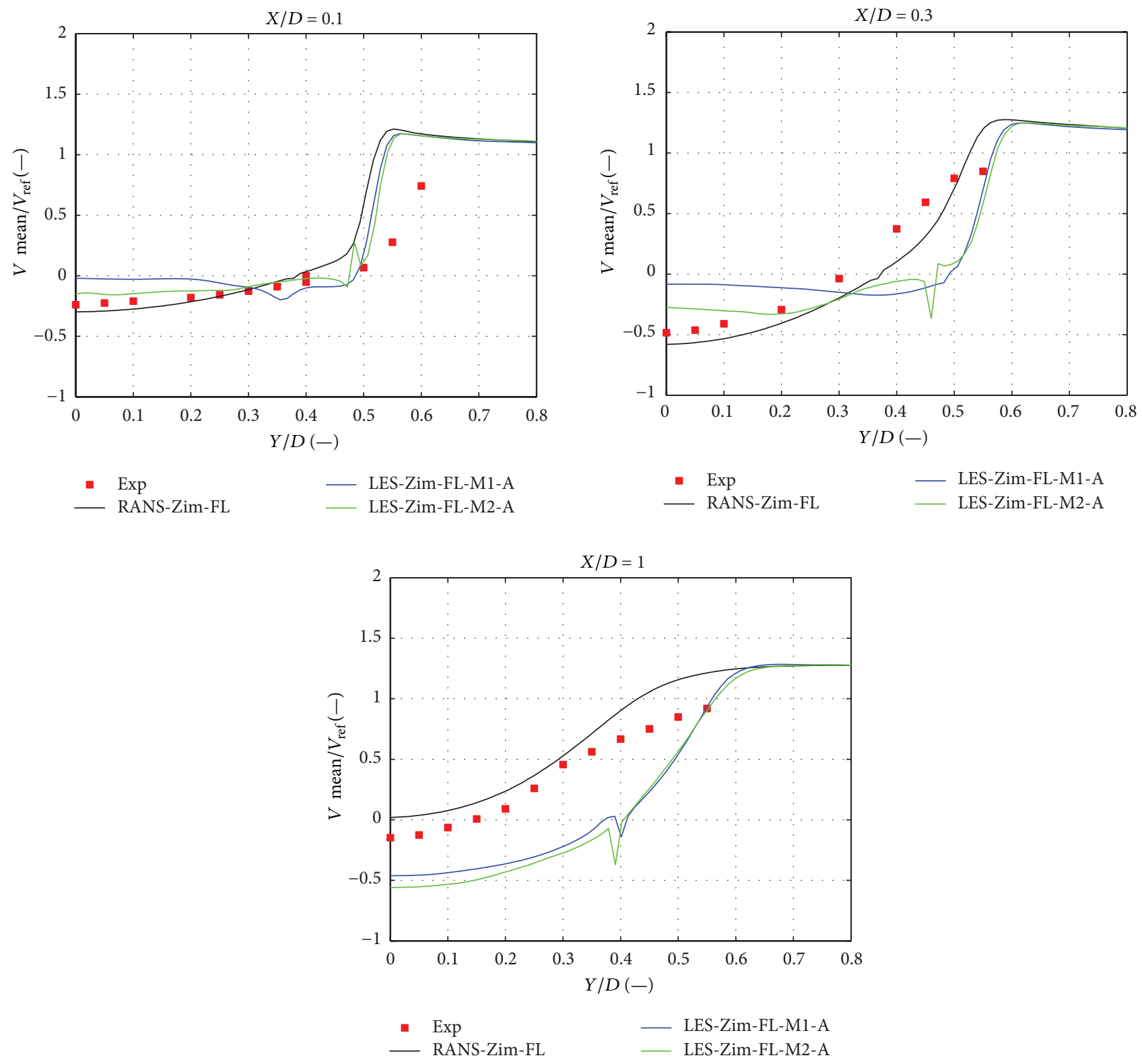

FIgURE 11: Mean axial velocity profiles at different axial locations.

mean temperature profiles as it is possible to observe from Figure 3.

A time step sensitivity analysis has been conducted using mesh B (Table 2) which is the best compromise between calculation time and solution accuracy. The time step needs to be small enough to catch the characteristic time of smallest structures [3]. Three time steps have been chosen $\left(6.5 e^{-5} \mathrm{~s}\right.$, $6.5 e^{-6} \mathrm{~s}$, and $\left.6.5 e^{-8} \mathrm{~s}\right)$. The longest was found not suited as it did not satisfy what was mentioned just above. This effect can be appreciated even looking at the mean temperature profiles in Figures 3 and 4. From the same figures can be observed how the two time steps $<1 e^{-5} \mathrm{~s}$ lead to the same result as they allow catching the smallest structure fluctuations. A time step of $6.5 e^{-6}$ s has been then chosen to continue the study, confirming the findings by other authors [3]. More detailed results on this preliminary quasi-2D study can be found in $[8]$.

5.2. Fully 3D Calculations. After the mentioned preliminary study, fully 3D calculations have been performed. The geometry (Figure 5) reproduces the experimental device. Two levels of mesh refinement have been realized and tested, guaranteeing in both cases a refinement in the region of the shear layer. The coarse mesh was not sufficiently refined to satisfy the above mentioned mesh criteria everywhere in the first part of the flame zone, while the fine one did.

On both meshes, the effects of an increase (doubling) in the constant $A$ in (3) have been evaluated. Table 2 reassumes the setup of the performed runs. 

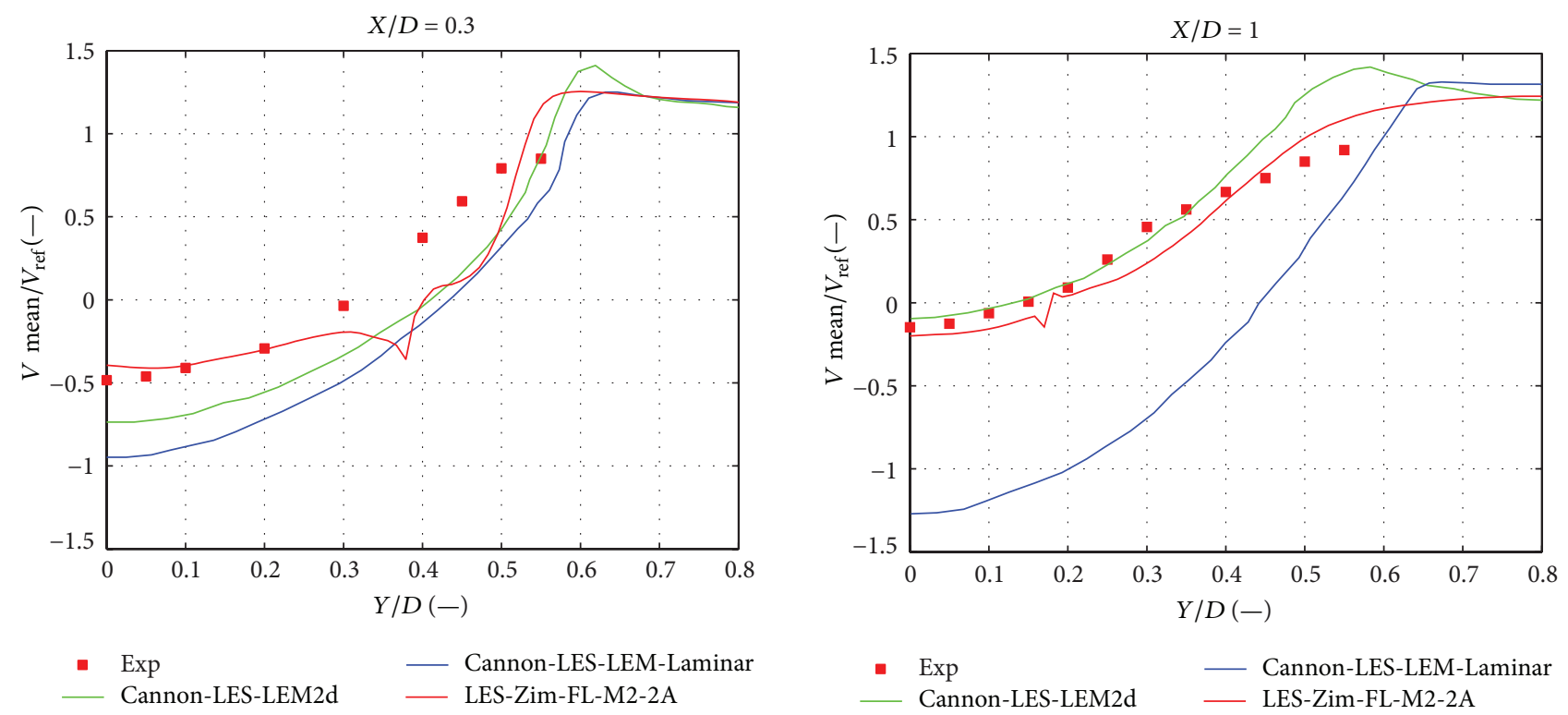

FIgURE 12: Mean axial velocity compared with Cannon et al. results [3].

5.3. Upstream Flow Conditions. Before discussing the flame behavior downstream the bluff body, it is due to underline some aerodynamics features observed in the feeding duct. In particular, turbulence intensity levels obtained in the bluff body throat section for the performed simulations are compared against experimental one.

All the simulations predict lower free turbulence intensity levels than the measured 24\%: RANS simulation reaches a TU of $13 \%$ while Fluent and OpenFOAM LES show a reduction of the provided inlet value up to $9 \%$ and $11 \%$, respectively. Such TU is computed for LES sampling and averaging velocity fluctuations to evaluate time-averaged resolved turbulent kinetic energy and adding the subgrid contribution. The low turbulence levels reached in all the cases might have a direct influence on the solution leading to local underestimation of reaction rates or missing quenching effects induced by flame stretch due to locally high turbulence levels in the cases when such effect is included in the simulation, that is, LES-Zim-FLM2-2A.

5.4. Flame Structure. Results obtained with Fluent LES are presented below describing the main features of the predicted flame. The influence of a mesh refinement as well as the mentioned change in the combustion submodel constant $A$ on the turbulence flame interaction and on the local burning rate is described together with a possible explanation about the resulting behavior and the differences emerged in the comparisons with the experimental data.

From progress variable contours at three different axial locations in Figure 6 emerges how the flame remains confined in the central part of the domain for the major part of the combustor length, while the external squared duct does not significantly affect the flame shape. The effect of the increased resolution cannot be appreciated in the first part of the burner, close to the bluff body, but becomes more evident downstream at $X / D$ larger than 1 . The flame is more wrinkled due to the small turbulent structure which were filtered out by the coarse mesh.

This behaviour affects the PFR that is increased close to the bluffbody, see Figure 8, as well as in the central part of the combustor, Figure 7. Such a change is not sufficient to complete combustion at the outlet nor to significantly change the mean progress variable contours. From mean temperature profiles in Figure 9 a slight difference is observed at $X / D=6$ section but the level predicted underestimates anyhow the measured temperature.

In all these cases the recirculation length is overpredicted (refer to Figures 10 and 11) with respect to RANS simulation and experimental results.

Changing the combustion model constant $A$ to 1.04 allows an excellent agreement with experimental results in terms of mean temperature and velocity profiles as depicted in Figures 9 and 11. The flow field and the recirculation length are well predicted. Such a change in $A$ constant boosts the combustion since the very first part of the bluff body end and the flame tends to move upstream the bluff body itself. To avoid flame flashback, critical strain rate limit is set to a lower value $\left(20000 \mathrm{~s}^{-1}\right)$, thus introducing local flame quenching in the regions of high stretch. The flame is enlarged and PFR increased (Figure 8). The result is to change the shear layer interactions between the fresh mixture and burnt gasses intensifying both turbulent mixing and chemical reactions. Being the equations coupled, an increased reaction turns into higher velocities in the second part of the domain and a shortening of the recirculation zone.

From instantaneous progress variable contours in Figure 7, it is possible to see how changing the model constant does not alter the flame wrinkling. The flame remains confined in the central part of the duct maintaining a hot core for the greatest part of the combustor length. Unlike the other LES results towards the outlet, at $X / D=6$ section, fully burnt gasses are now obtained. 

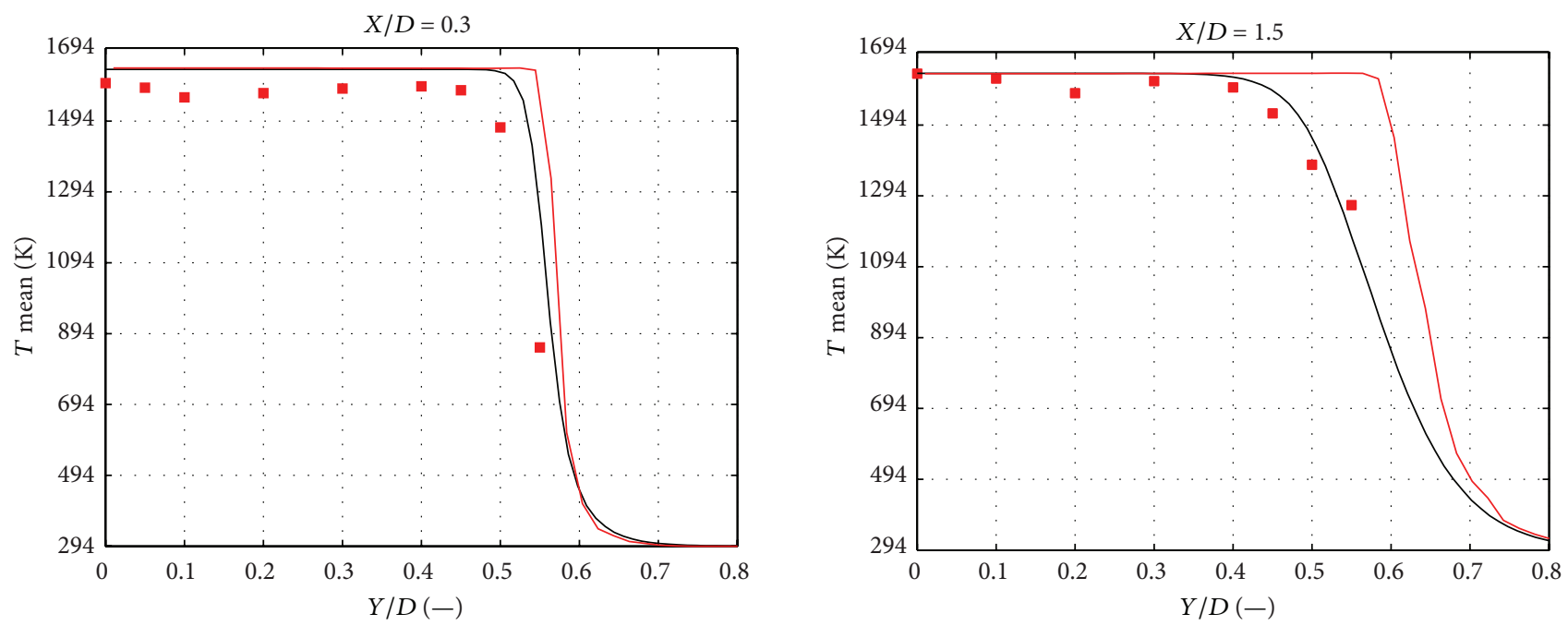

- Exp

- RANS-Zim-FL

- Exp

- RANS-Zim-FL

— URANS-Xi-OF
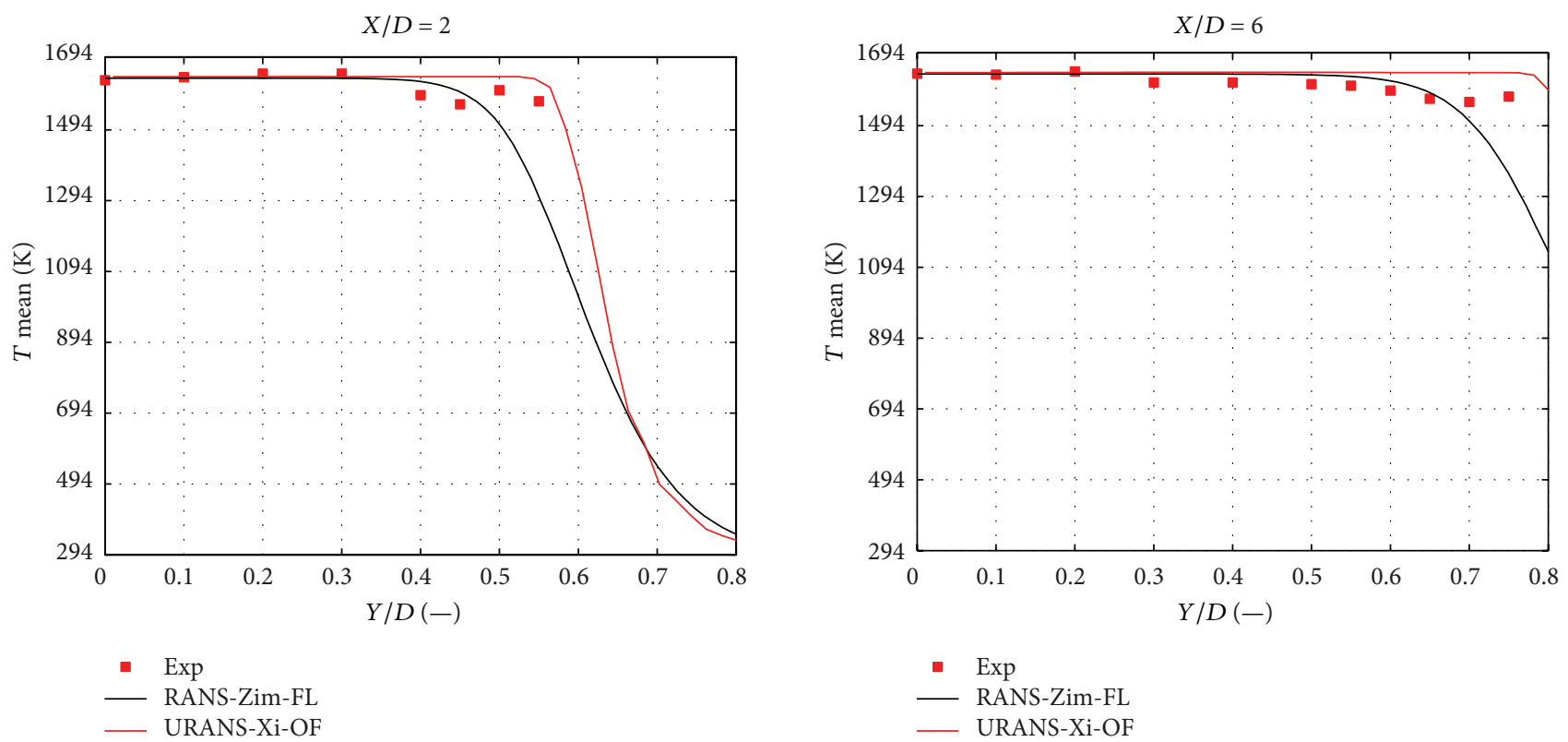

FIGURE 13: Mean temperature profiles with OpenFOAM (URANS).

Looking at mean progress variable contours predictions with LES-Zim-FL-M2-2A lead to a significantly different $c$ levels when compared to other LES simulations and the results are closer to the RANS one. With RANS the flame does not attach to the duct wall but in the converging part, while in LES-Zim-FL-M2-2A mean progress variable contours show that flame anchoring is achieved on the straight duct.

Temperature profiles from simulations performed by Cannon et al. [3] are included for reference in Figure 9. It refers to a Large Eddy Simulation coupled with a linear eddy model (LES-LEM), used to describe the sub-grid chemistry and turbulence-chemistry interactions, using a single-step mechanism, performed on a fully 3D test case. Figure 9 shows how the LES-LEM nicely predicts temperature profiles even though they are available only at $X / D=0.3$ and 6, whereas also case LES-Zim-FL-M2-2A presents a quite good agreement with measurements. The effect of LEM is to enhance the filtered reaction rate due to sub-grid turbulent fluctuations, substantially acting in the same way of the modified $A$ constant in Zimont model. This may suggest that a much more refined grid or a different subgrid scale model ought to be used when performing LES simulation in order to catch the smallest scale effect on reaction rates. Both these solutions have the drawback of a large increase in calculation time.

A general overprediction of temperature levels in the recirculation zone by the computational models is found in all the studied cases (Figure $9 X / D=0.3$ ). This effect is mainly 

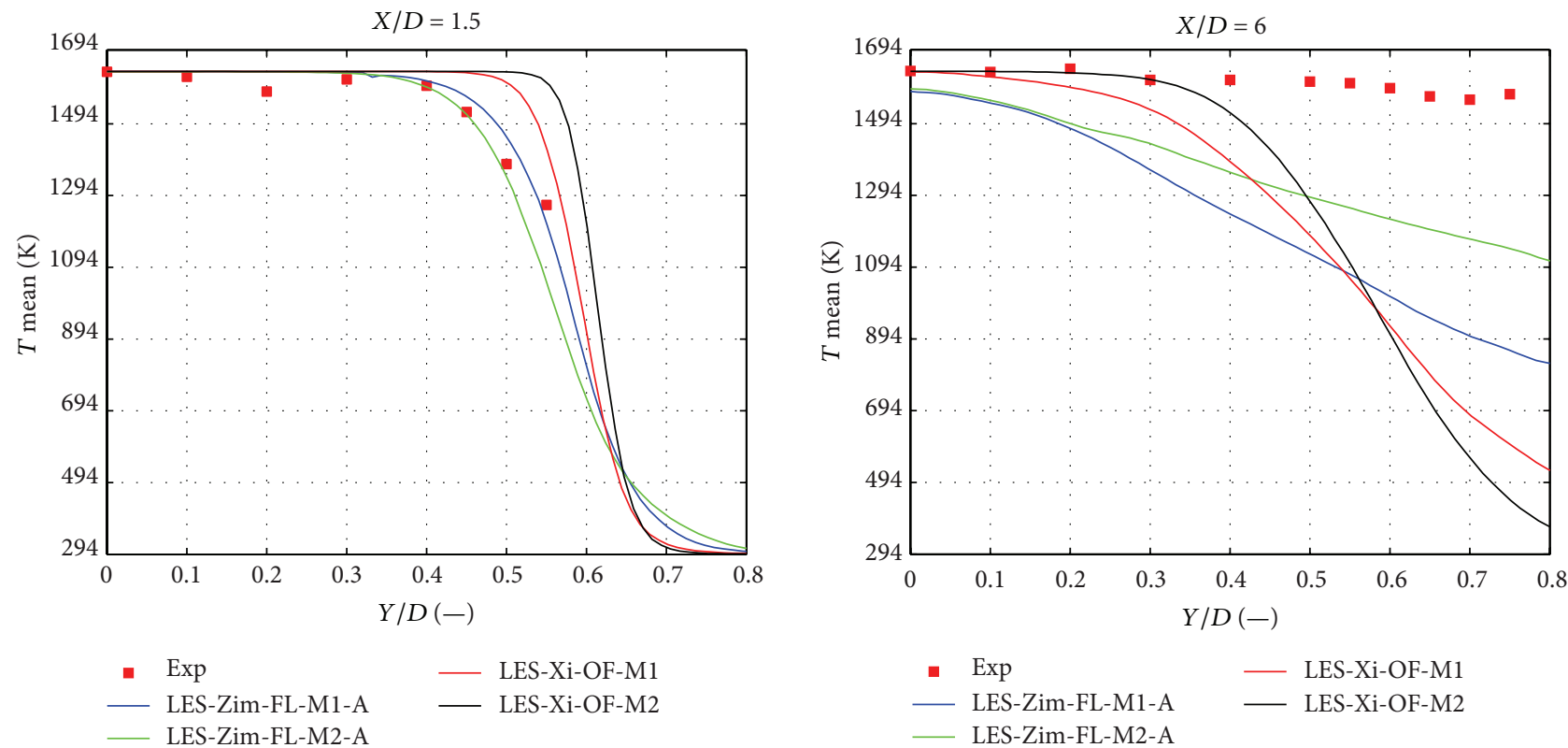

-
Exp
LES-Zim-FL-M1-A

— LES-Xi-OF-M1

- LES-Zim-FL-M2-A

- LES-Xi-OF-M2

FIGURE 14: Mean temperature profiles with OpenFOAM and Fluent (LES).
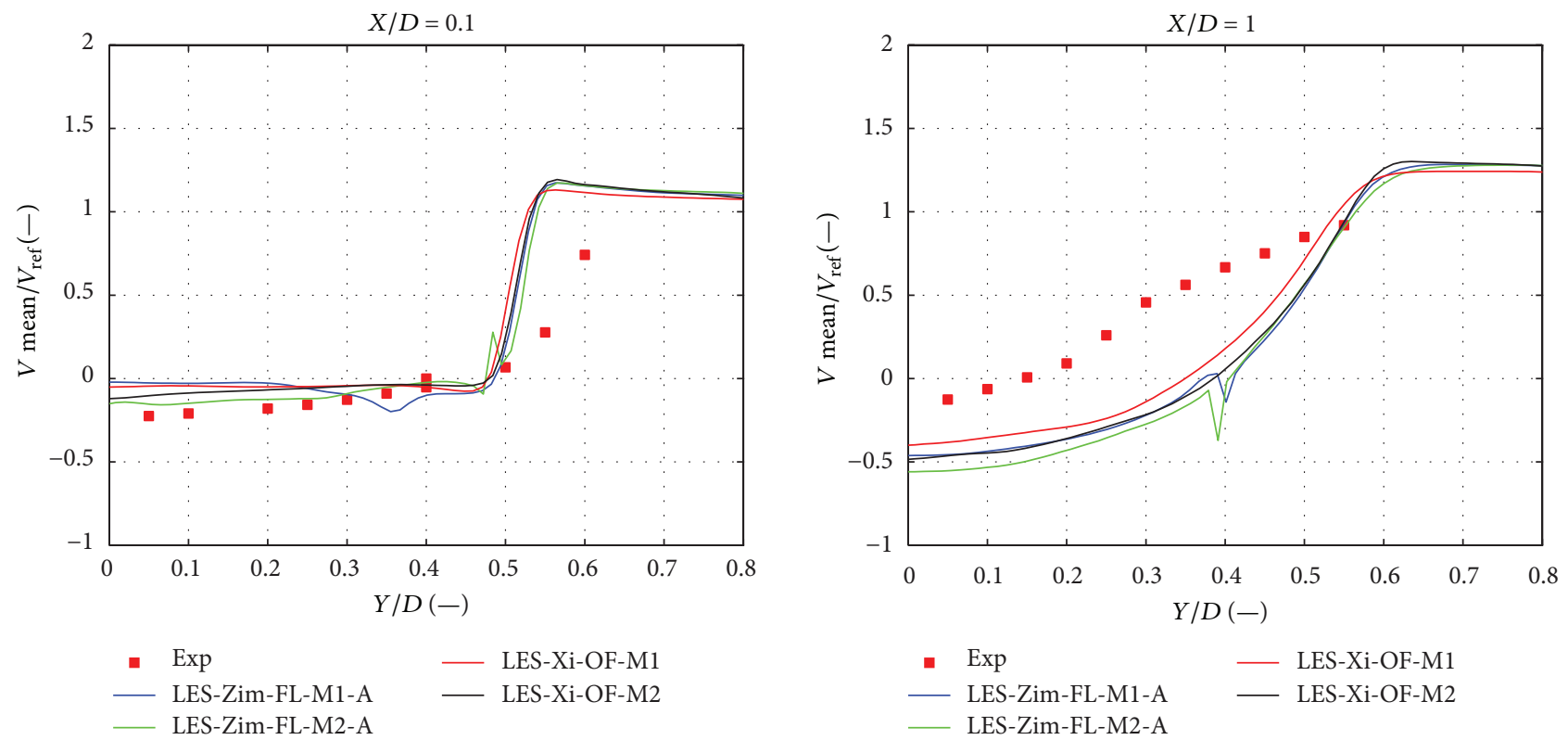

FIGURE 15: Mean axial velocity profiles with OpenFOAM and Fluent (LES).

due to the thermal boundary condition on the bluffbody wall which in the computations is considered adiabatic. Nandula [10], in fact, estimates a heat loss at the bluffbody walls of around 6\%. A fair inappropriate prediction of aerodynamic stretch may also locally influence the accuracy [15]: this is confirmed by recalling that implemented TFC model does not account for flame stretch giving an explanation to the underestimated mean flame brush thickness and to the higher values of flame temperature predicted by the model.

Another common aspect, highlighted in Figure 10, is the overprediction of the flame length respect to experiments.
As already pointed out, at $X / D=6$ only LES-Zim-FL-M2$2 \mathrm{~A}$ succeeds in achieving a completely developed and burnt flame while the reaction is not complete in all the other cases. This discrepancy also affects the upstream sections. Looking at temperature profiles at $X / D=1.5$ and $X / D=3$ in Figure 9, the observed flame brush is thinner, with sharp gradient, characteristic of a delayed profile which has not conveyed the fresh gases entrainment and diffusion. Such effect tends in fact to reduce when the total flame length is well predicted.

The regions more sensitive to an increase in turbulence levels are the reaction zones downstream of the recirculation 


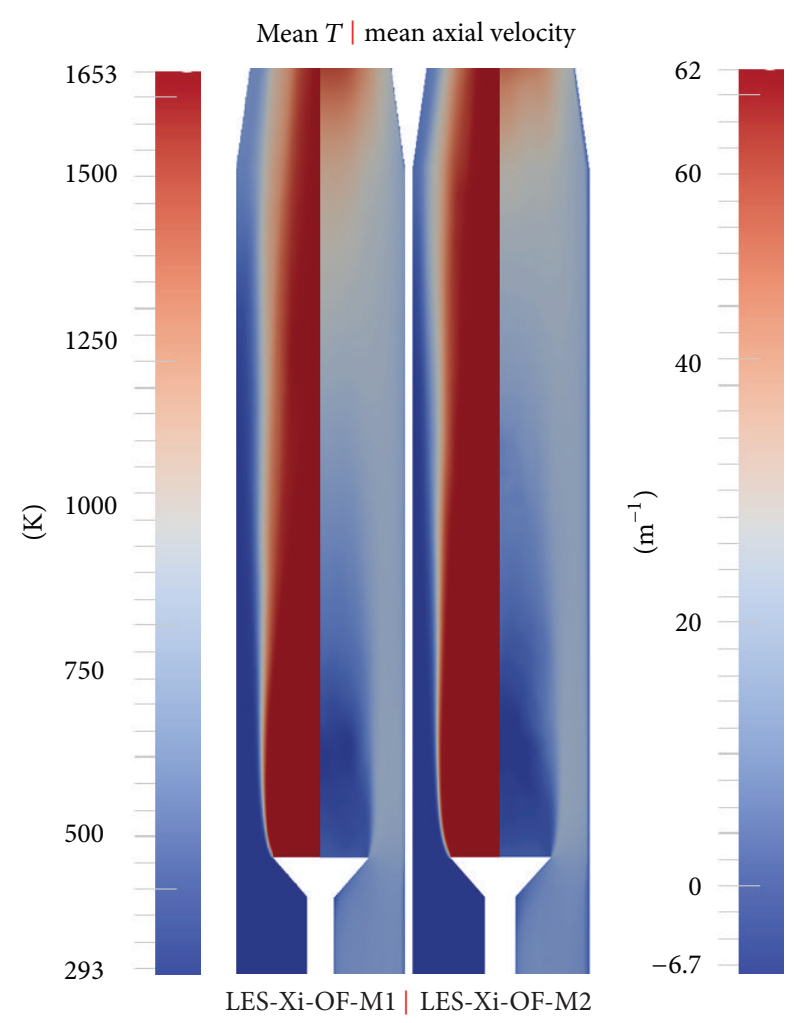

Figure 16: Mean temperature and axial velocity with OpenFOAM (LES).

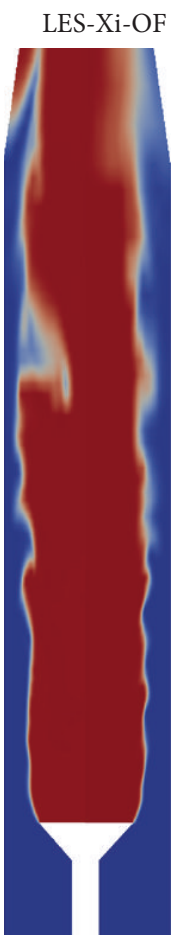

M1 | M2

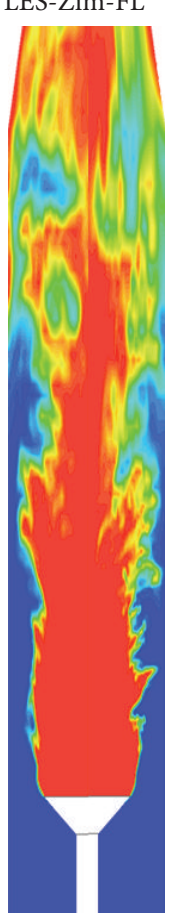

M1 | M2
FIGURE 17: Instantaneous temperature field with OpenFOAM and Fluent (LES). zone. In fact the flame is not supported by the hot products of the recirculation zone, but it behaves as a freely propagating flame in that region. The measured strain rates are lower and a thin flame (shear layer) begins its growth. This shear layer (reaction zone) thickens as flame travels downstream due to entrainment of fresh reactants and large-scale coherent structures fold around the flame edge [10]. The effect of an increase in the modeling constant $A$ in (3) is to partially compensate the lower turbulence level (lower $\mathrm{u}^{\prime}$ ) thus leading to profiles closer to the experimental ones.

In the axial velocity plots shown in Figure 12, two results from Cannon et al. [16] are presented together with experimental and LES-Zim-FL-M2-2A results. In this study they used a laminar chemistry LES model and a LES-LEM with 1-step mechanism, both performed on a $2 \mathrm{D}$ test case. LESLEM of Cannon shows a good agreement with experimental results while the LES with laminar chemistry overpredicts the recirculation zone length. This confirms how an underpredicted reaction rate leads to a different flow field with a longer recirculation zone and how increasing the model constant may lead to satisfying results as well as a more onerous subgrid scale modeling approach.

5.5. OpenFOAM LES Results with Xi Closure. In order to generate a realistic initial condition and provide more comparable references for the LES results, URANS was also performed. Comparing results and measurements in Figure 13, it may be observed that the temperature is overpredicted in the first part of flame while fits well experimental data in the downstream sections. Towards the outlet, the combustion is complete and the flame tends to reach the ducts wall slightly before than in Fluent RANS solution.

LES simulation results, presented in Figure 14, suggest the same trend highlighted by the RANS calculations that is the quite sharp mean flame front close to the bluff body positioned at higher radii compared to both experiments and calculations with Fluent and the incomplete level of burnt gases even at the end of the flame tube. This incomplete combustion is however partially reduced with the finer mesh showing temperature profiles more in agreement with the experiments at the outflow section than the equivalent computations performed with Fluent.

Also the retarded closure of the recirculation bubble compared to measurements is recorded for the OpenFOAM computations as for runs with unmodified Zimont constant, see Figure 15.

These findings may also be noted in Figure 16, where the time-averaged values of temperature and axial velocity are shown on a longitudinal plane.

However, it is important to verify the behavior of the flame also from a qualitative point of view, looking at the flame front shape to describe the regime of reaction. This can be done observing the instantaneous temperature fields in Figure 17 which depicts a less wrinkled and less diffused flame front compared to Fluent. parison between ANSYS Fluent LES and OpenFOAM native 

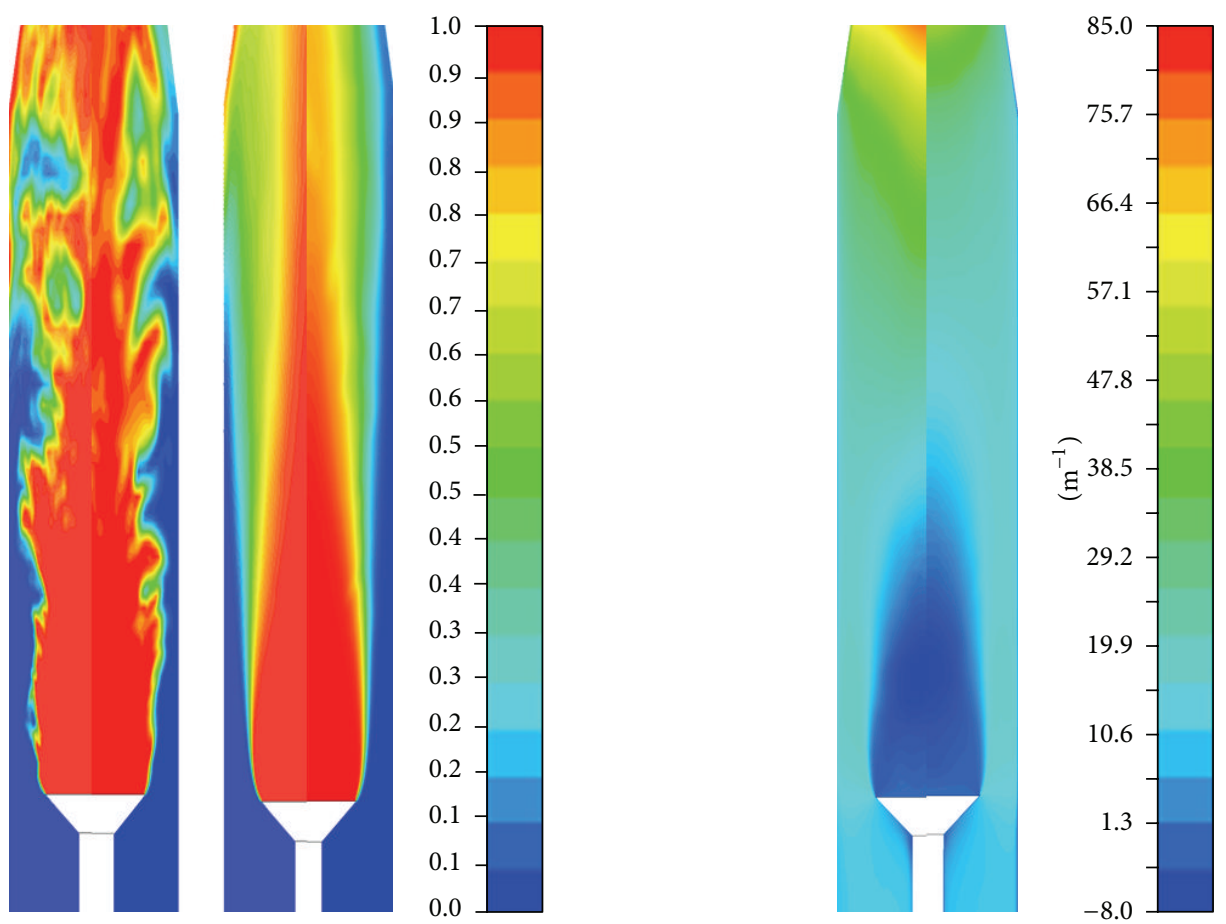

FIGURE 18: Instantaneous and mean progress variable and mean axial velocity contours for Fluent LES-ZIM-FL-M1-A and Zimont OF on mesh M1.

solver XiFoam LES was the initial aim of the work. However, obtained solutions predict different flame behaviors and turbulence-chemistry interactions. Having used different combustion submodels, it is not possible to know if obtained discrepancies are due to numerical aspects or to the combustion submodel itself. For a direct evaluation of the effects of the numerics implemented in the two codes, Zimont turbulent closure has been implemented in OpenFOAM to provide a solver nominally equivalent to that used in Fluent. Results of this analysis are presented here, comparing the two solutions in terms of predicted flame features, mean temperature, and mean velocity profiles, highlighting the main numerical aspects which might influence the solution.

When Zimont closure is used in OpenFOAM, obtained results are in line with Fluent ones as demonstrated by the test performed on M1. Looking at Figure 18, it is possible to observe that the two flames show the same features: flame front wrinkling is characterized by equivalent length scales with large vortices entraining the hot core for $X / D>2$. Some discrepancies however emerge between the two cases: from mean progress variable contours it is evident that the Zimont OF M1 flame is not able to attach the duct wall. This effect may be due to the different near wall treatment implemented in the subgrid model which results in a lower velocity close to the wall for Fluent calculation which helps stabilize the flame to the wall. The missed wall attachment of the flame in the final part of the combustor induces an intermittent flame detachment in OpenFOAM. The main differences in mean progress variable contours can be appreciated in the central part of the combustor, where the mean flame front angle is higher in LES-ZIM-FL-M1-A and even more from the point where this latter simulation predicts an attached flame on the wall. The effect is visible also in the temperature profiles in Figure 20 at the last sampled section $X / D=6$ where the reaction progress, and in turn temperature which is calculated according to (1), does not reach the same values predicted by Fluent LES.

From both mean $c$ contours and temperature profiles, it is possible to see that OpenFOAM model predicts a thicker flame brush. This is probably due to the filtered linear discretization scheme exploited for regress variable $b$ equation which is an extension of the purely linear scheme with a filter for high-frequency ringing [17]. This filtering operation acts in the same direction of a mesh coarsening (see i.e., M1-M2 differences) and has an impact on local turbulent flame speed $\left(S_{t}\right)$ values and in turn on the flame brush; see Figure 19. The reaction to be completed (progress variable from 0 to 1) needs a larger space (brush). Looking at PFR contours in Figure 19, being the product between $S_{t}$ and $c$ gradient, results in lower peak values, due to smoothed $c$ gradients but with nonzero values over a larger zone. Reaction progress variations are in fact spread over a longer distance. From temperature profiles shown in Figure 20, a general agreement is, however, found for all the other sections.

The resulting flow field is consistent with the one predicted by Fluent. Higher velocity are predicted by the latter (Figure 18); the effect is due to the previously discussed phenomena of intermittent flame detachment which lead to intermittent density variation, gas expansion, and mean velocity. From profiles in Figure 21, a general agreement is observed in the velocity field predicted by the two codes. 


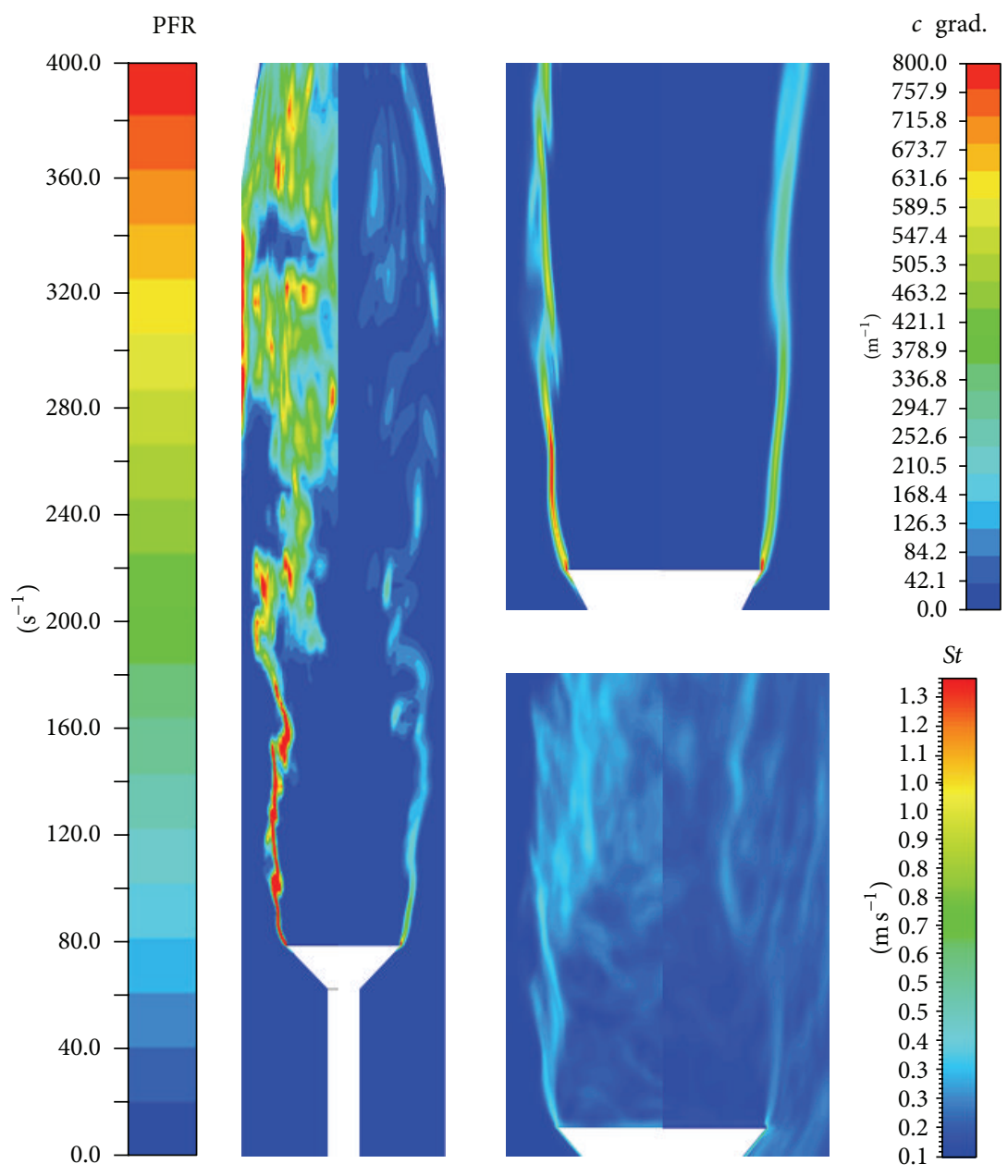

FiguRE 19: Product formation rate, turbulent flame speed, and progress variable gradient contours.

5.7. Calculation Time Considerations. In order to provide a guideline for future works, it has been retained useful to evaluate and compare calculation times between the two codes and to provide an estimate of the computational overhead connected with LES compared to RANS and its increase due to the need of a larger number of time steps and a more refined mesh level.

In this case where RANS can exploit 2D assumptions the additional computational resources needed for LES become relatively enormous: almost 100 times.

Comparison of CPU time is hence conducted for LES only on simulations performed on the coarsest mesh (915000 elements). The global computational time is computed as function of the time needed to complete a time step and the total number of time steps employed by the solution to stabilize. In this analysis, the employed time step is equal for all computations so the total number of iterations to cover the same amount of physical time is the same. However, depending on the obtained regime of flame, it is necessary to extend the averaging time to stabilize mean quantities on different time periods.

In terms of computational efficiency per single time step, OpenFOAM overwhelms Fluent with a time ratio of approximately 0.15 due to the different time advancement algorithm which minimizes the number of inner iterations. This ratio is almost independent by the type of combustion modeling (closure following [6] or [2]) employed. The additional time related to the use of $\mathrm{M} 2$ is approximately $30 \%$ for OpenFOAM and $150 \%$ for Fluent. However, as already hinted, the total number of iterations needed to reach smooth and stable timeaveraged profile is quite different between the considered cases. Computations with Zimont closure showed that only 8000 time steps were necessary for Fluent while more than 90000 time steps were computed with OpenFOAM. This large difference is principally due to the low frequency of flame detachment from the wall.

\section{Conclusions}

LES simulations of a bluff body stabilized lean premixed flame have been performed using both a commercial and an open-source CFD code. Preliminary analysis conducted on a quasi-2D test permitted assessing the influence of local mesh resolution and computational settings as well as providing a reference RANS solution which was exploited to evaluate the effective computational cost increase and the physical insight enhancement associated with LES compared to standard modeling practices. 

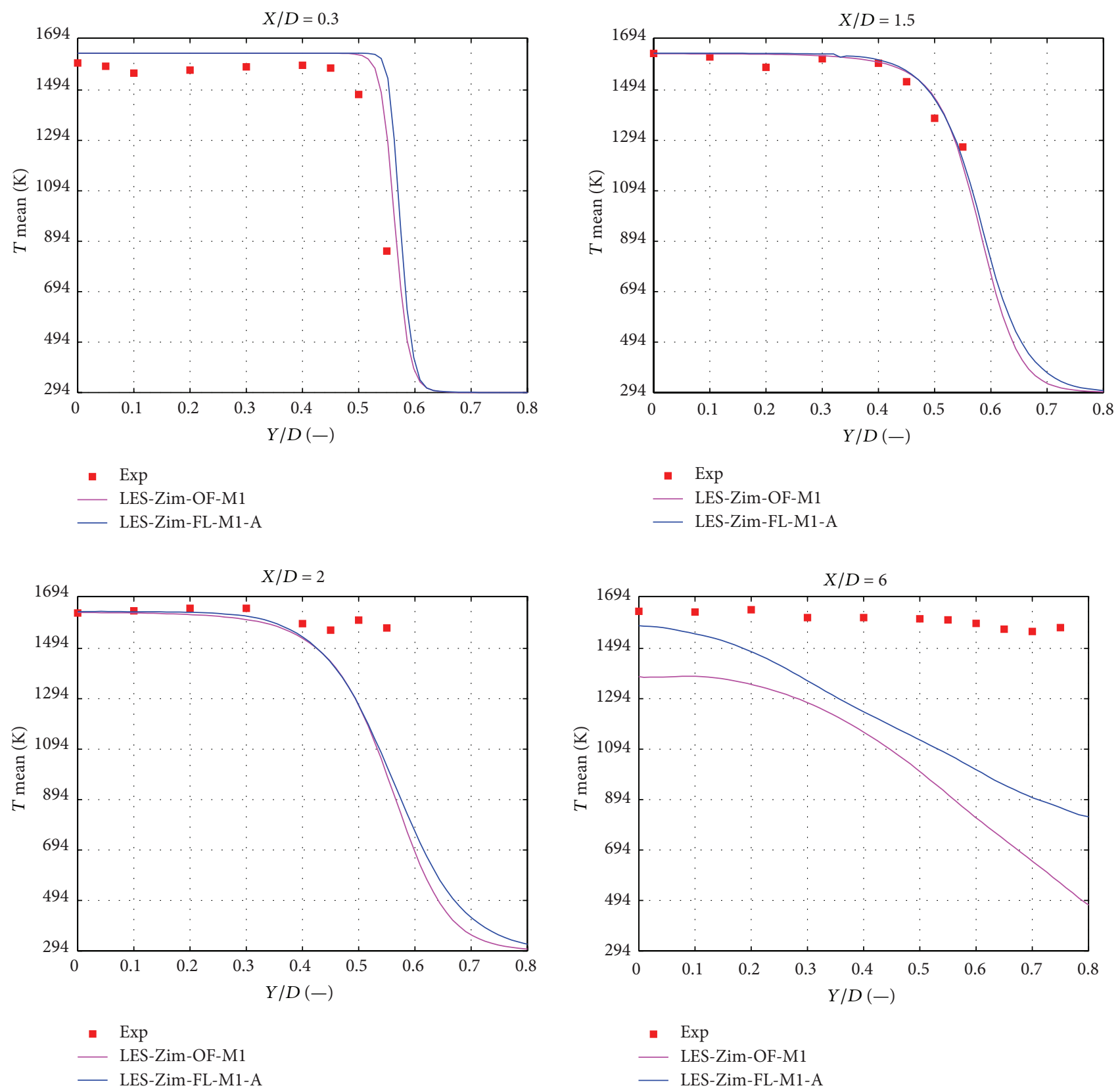

Figure 20: Temperature profiles for Fluent LES-Zim-FL-M1-A, experimental results, and LES-Zim-OF-M1.

LES performed with Zimont turbulent flame speed closure show that if the grid clustering is based on aerodynamic and RANS flame thickness considerations, it is necessary to tune model constant $A$ to achieve a complete combustion at the exit of the burner. Solutions obtained with Fluent and OpenFOAM using nominally same models are equivalent in terms of mean profiles at least up to $X / D<2$. Main differences are limited to the near wall flame behavior and the flame brush thickness which are, respectively, due to the employed wall functions and the advective interpolation scheme for the progress variable equation.

Native OpenFOAM closure based on Xi transport equation predicts an even thinner flame brush associated with a very low wrinkling resulting in limited interaction between fresh and burnt gases downstream the bluffbody and in reduced combustion progress in the region at larger radii for $X / D>3$.

In terms of computational resources, OpenFOAM requires a much smaller time to complete a single time step; however in the only case of equivalent physical modeling performed (M1 with Zimont closure), the higher number of time step necessary to achieve converged mean solution, due to a more unsteady behavior of the flame, resulted in equivalent global computational efforts.

RANS calculations succeed in predicting mean values with substantially equivalent accuracy and considerably 

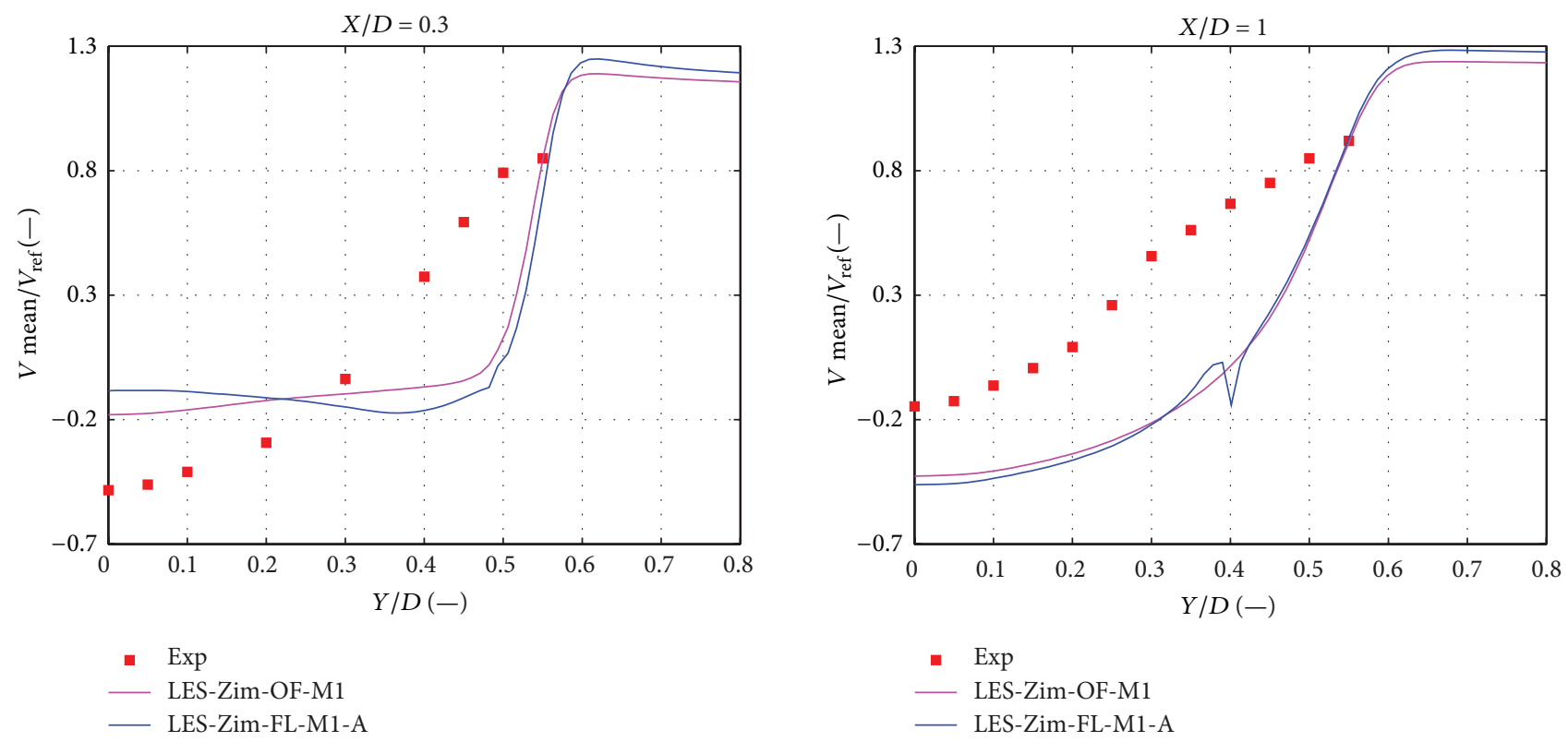

FIGURE 21: Velocity profiles for Fluent LES-ZIM-FL-M1-A, experimental results, and Zimont OF.

lower calculation time $1 \%$. LES potential thus may be identified in providing more accurate and detailed resolution of the field of motion and better capture the turbulencechemistry interaction. This is however reflected in more accurate prediction of the flame shape only after a long tuning process of the computational setup.

The large computational resources required for LES would be compensated by really improved, qualitatively and quantitatively, results, only when applied to complex cases, such as swirled flames or complete combustor geometry where RANS models fail in accurately predicting the flame stabilization process as well as the flame anchoring.

As far as RANS simulations are concerned, more accurate results on the studied test case might be obtained exploiting a turbulent combustion model able to properly predict the flame length and which takes into account chemical species as well as nonequilibrium effects on these latter, due to the interaction with turbulent flow field. Possible models to be used are $G$-equation model, which has been shown to provide good agreement with experimental data [15], or flamelet generated manifolds in both RANS and LES context, being their LES implementation straightforward.

\section{Conflict of Interests}

The authors declare that there is no conflict of interests regarding the publication of this paper.

\section{References}

[1] V. Raman and H. Pitsch, "Large-Eddy Simulation of a bluffbody-stabilized non-premixed flame using a recursive filterrefinement procedure," Combustion and Flame, vol. 142, no. 4, pp. 329-347, 2005.
[2] V. Zimont, W. Polifke, M. Bettelini, and W. Weisenstein, "An efficient computational model for premixed turbulent combustion at high Reynolds numbers based on a turbulent flame speed closure," Journal of Engineering for Gas Turbines and Power, vol. 120, no. 3, pp. 526-532, 1998.

[3] S. Cannon, B. Zuo, V. Adumitroaie, and C. Smith, Linear Eddy Subgrid Modeling of a lean Premixed methane-Air Combustion, CFDRC Report 8321/8, 2002.

[4] T. Poinsot and D. Veynante, Theoretical and Numerical Combustion, Edwards, 2001.

[5] Fluent Vers. 14. 0 Theory Guide.

[6] H. G. Weller, G. Tabor, A. D. Gosman, and C. Fureby, "Application of a flame-wrinkling LES combustion model to a turbulent mixing layer," Symposium (International) on Combustion, vol. 27, no. 1, pp. 899-907, 1998.

[7] A. Yoshizawa, "Bridging between eddy-viscosity-type and second-order models using a two-scale DIA," in Proceedings of 9th Symposium on Turbulent Shear Flows, 1993.

[8] S. Menon, P. K. Yeung, and W. W. Kim, "Effect of subgrid models on the computed interscale energy transfer in isotropic turbulence," AIAA Paper, pp. 2394-2378, 1994.

[9] H. Lugt, Introdution To Vortex Theory, Potomac, Vortex Flow Press, 1996.

[10] S. P. Nandula, Lean Premixed Flame Structure in Intense Turbulence Rayleight/Raman/LIF Measurements and Modeling [Ph.D. thesis], Faculty of the Graduate School of Vanderbilt University.

[11] N. Peters, Turbulent Combustion, Monographs on Mechanics, Cambridge, Mass, USA, 2000.

[12] M. H. Baba-Ahmadi and G. Tabor, "Inlet conditions for LES using mapping and feedback control," Computers and Fluids, vol. 38, no. 6, pp. 1299-1311, 2009.

[13] A. Innocenti, Large Eddy Simulation of a Bluff Body Stabilized Lean Premixed Flame [M.S. thesis], University of Florence, 2011.

[14] L. Vervisch, P. Domingo, G. Lodato, and D. Veynante, "Scalar energy fluctuations in Large-Eddy Simulation of turbulent 
flames: statistical budgets and mesh quality criterion," Combustion and Flame, vol. 157, no. 4, pp. 778-789, 2010.

[15] M. Cerutti, A. Andreini, B. Facchini, and L. Mangani, "Modeling of turbulent combustion and radiative heat transfer in a object-oriented CFD code for gas turbine application," in Proceedings of the ASME Turbo Expo, pp. 809-822, June 2008.

[16] S. Cannon, V. Adumitroaie, and C. Smith, LES Software for the Design of Low Emission Combustion Systems For Vision 21 Plants, CFDRC Report 8321/7, 2002.

[17] OpenCFD, OpenFOAM User Guide. 

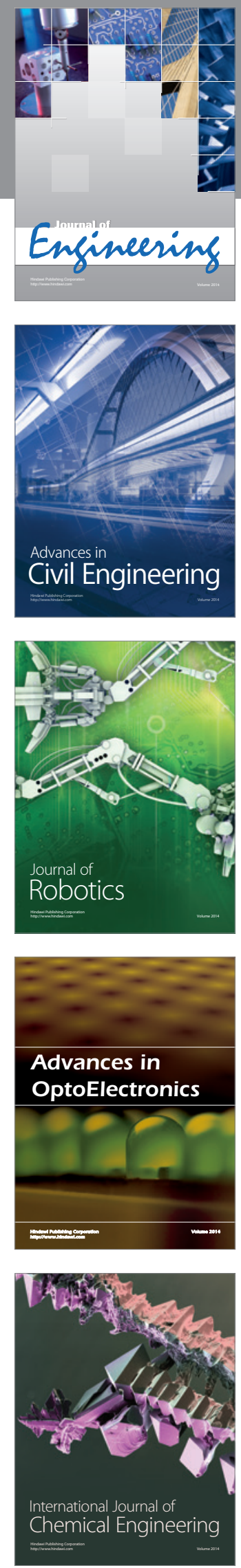

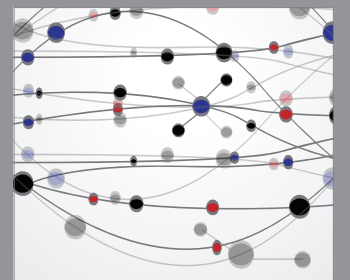

The Scientific World Journal
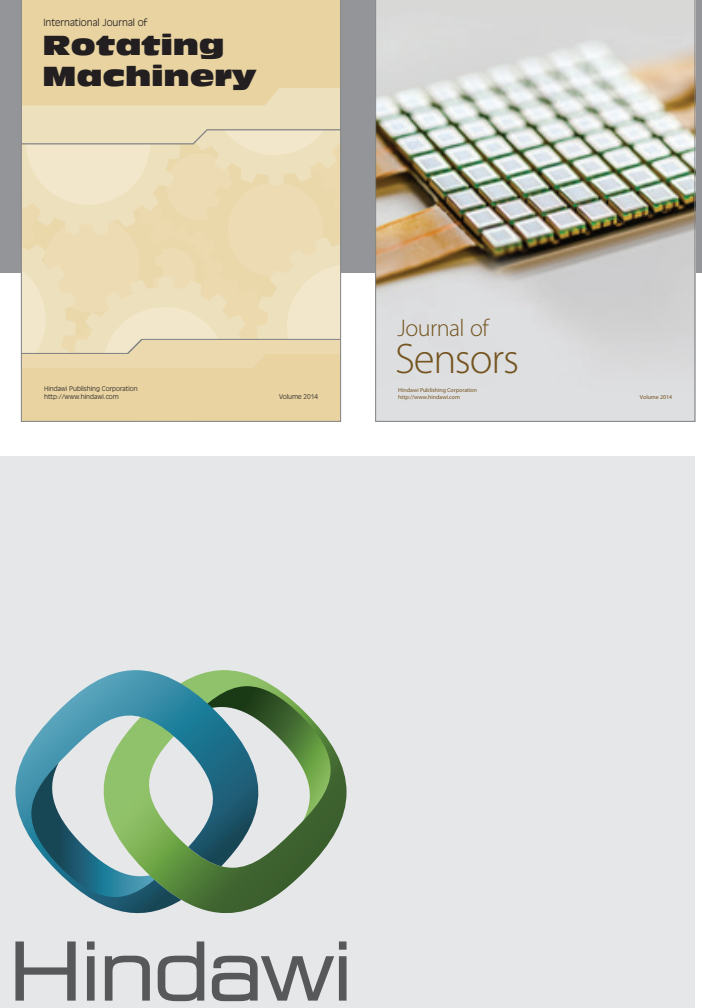

Submit your manuscripts at http://www.hindawi.com
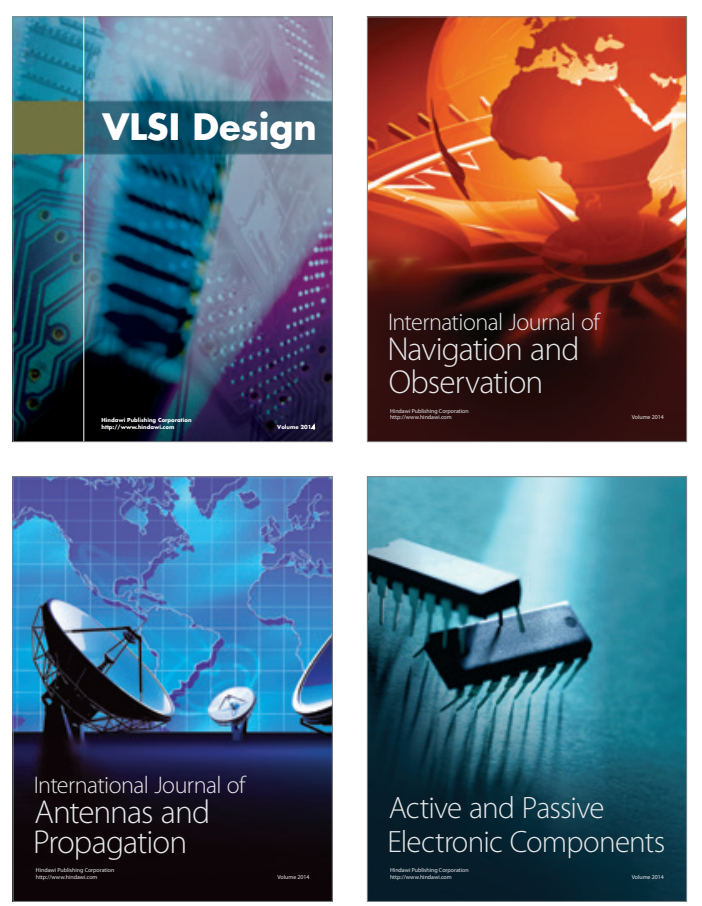
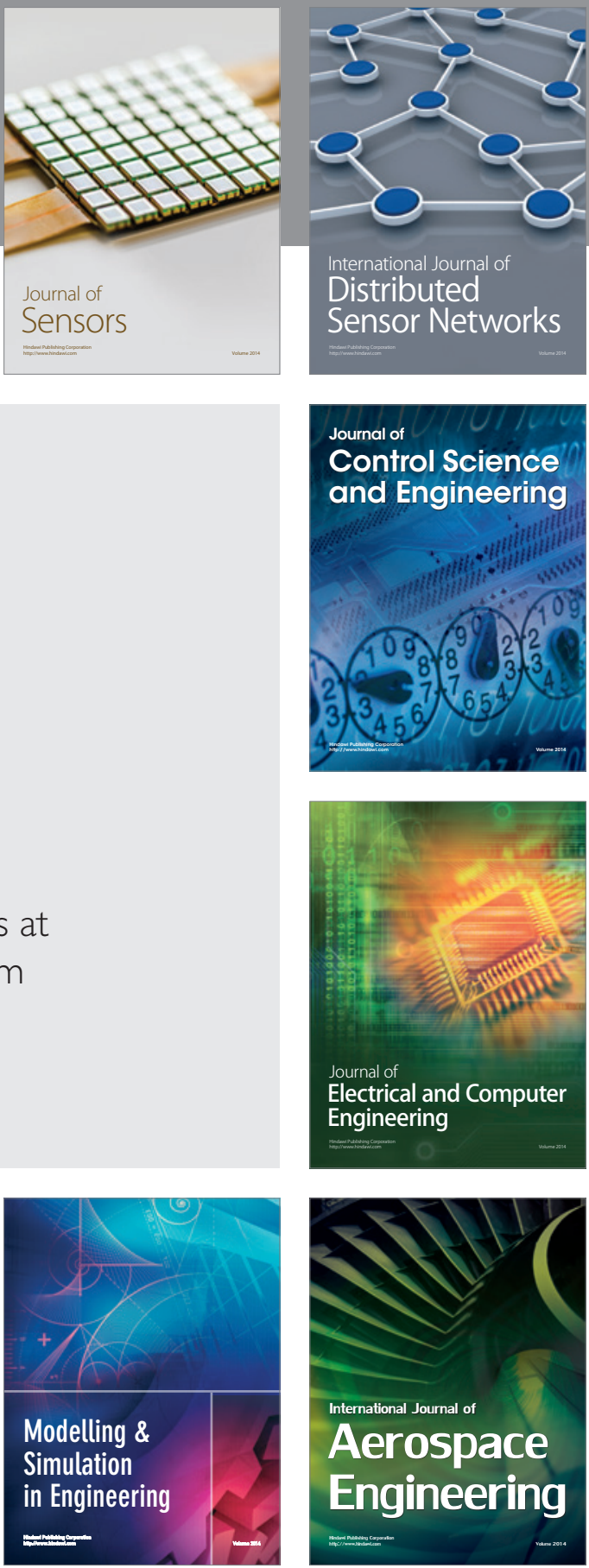

Journal of

Control Science

and Engineering
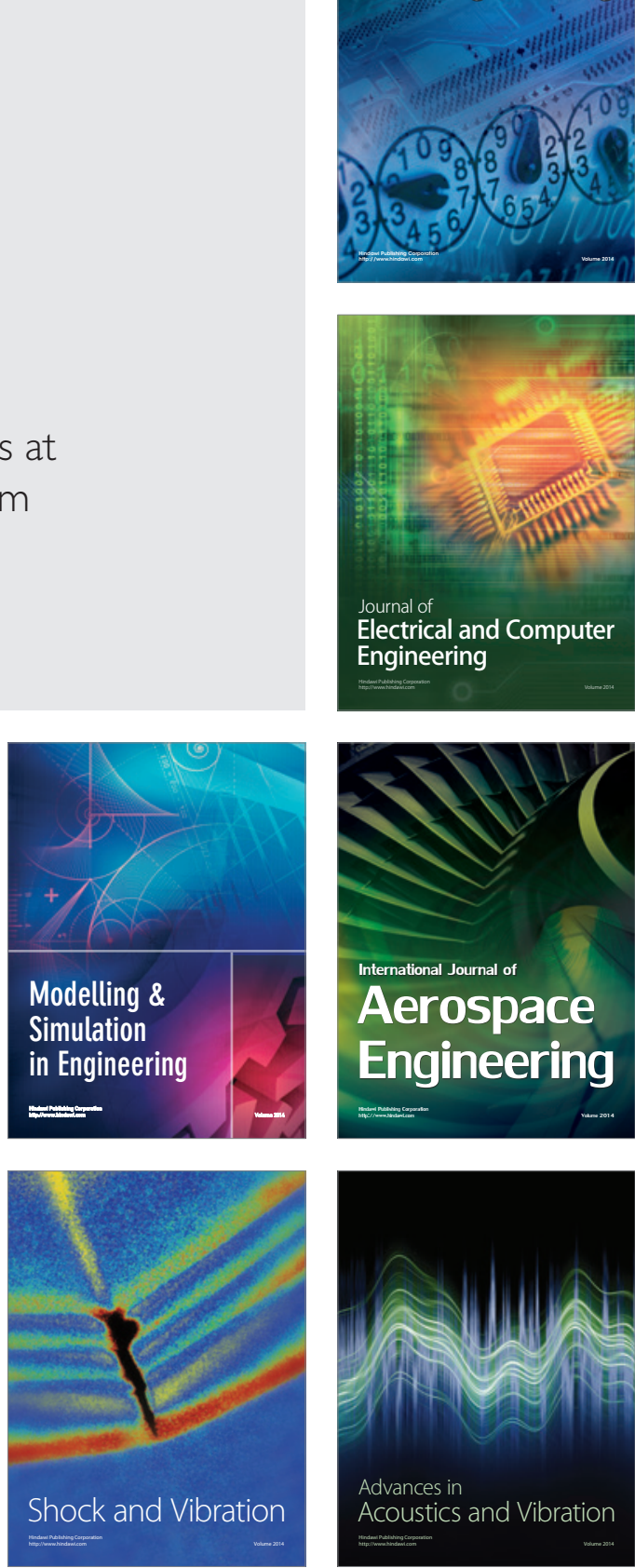